San Jose State University

SJSU ScholarWorks

Master's Projects

Master's Theses and Graduate Research

Spring 2011

\title{
User Monitor \& Feedback Mechanism for Social Scientific Study on Laptop Energy Reduction
}

Namrata Buddhadev

San Jose State University

Follow this and additional works at: https://scholarworks.sjsu.edu/etd_projects

Part of the Other Computer Sciences Commons, and the Systems Architecture Commons

\section{Recommended Citation}

Buddhadev, Namrata, "User Monitor \& Feedback Mechanism for Social Scientific Study on Laptop Energy Reduction" (2011). Master's Projects. 178.

DOI: https://doi.org/10.31979/etd.rb8n-ceu2

https://scholarworks.sjsu.edu/etd_projects/178

This Master's Project is brought to you for free and open access by the Master's Theses and Graduate Research at SJSU ScholarWorks. It has been accepted for inclusion in Master's Projects by an authorized administrator of SJSU ScholarWorks. For more information, please contact scholarworks@sjsu.edu. 


\title{
User Monitor \& Feedback Mechanism for Social
}

\section{Scientific Study on Laptop Energy Reduction}

\author{
A Research Project \\ Presented to \\ The Faculty of the Department of Computer Science \\ San Jose State University \\ In Partial Fulfillment \\ of the Requirements for the Degree \\ Master of Computer Science
}

by

Namrata Buddhadev

Spring 2011 
(C) 2011

Namrata Buddhadev

ALL RIGHTS RESERVED

ii 


\section{SAN JOSÉ STATE UNIVERSITY}

The Undersigned Project Committee Approves the Project Titled

USER MONITOR \& FEEDBACK MECHANISM FOR SOCIAL SCIENTIFIC

STUDY ON LAPTOP ENERGY REDUCTION

by

Namrata Buddhadev

APPROVED FOR THE DEPARTMENT OF COMPUTER SCIENCE

Prof. Mark Stamp, Department of Computer Science Date

Prof. Morris Jones, Department of Electrical Engineering Date

Prof. Robert Chun, Department of Computer Science Date 


\begin{abstract}
The dawn of the laptop era presents new challenges to the computing research community. Performance is no longer the end all be all of computing research because a new, significant requirement has cropped up: reducing the power consumption and improving battery usage. The key restriction to mobility is the constant need to be near a power outlet to recharge the laptop battery. Thus, extending battery life without compromising performance is a pressing concern.
\end{abstract}

A research named as "iGreen" was proposed to stimulate power consumption awareness and behavioral change among laptop users via interactive energy-usage feedback with college students as the initial target group.

This project is part of iGreen the project with an objective to analyze the users' behavioral changes by examining their usage of laptops and their attitude towards the environmental issues for energy consumption. It also tries to find reasoning and provide a social feedback mechanism to improve the efficiency of energy consumption and reduce the waste of power. 


\section{Acknowledgment}

Firstly, I would like to thank to Dr. Mark Stamp \& Prof. Morris Jones, my project advisors, for their guidance and support throughout my Masters' degree and project.

Secondly, I am highly indebted to Dean Belle Wei and Prof. Donald Hung for giving me chance to work on the research project. I thank them for the patience, ideas, suggestions and inspirations without which this research project would not have been possible.

Next I would like to thank my committee member, Dr. Robert Chun for helping me during the project and providing his guidance.

Lastly, this acknowledgment would be incomplete without thanking Jay Patel and Pankaj Sitpure for their constant help during research project. 


\section{Contents}

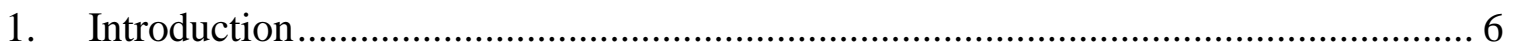

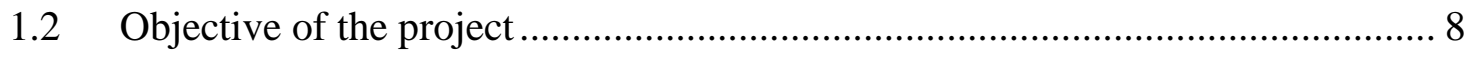

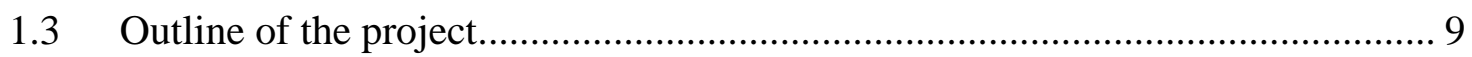

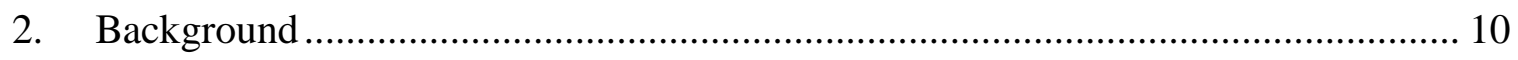

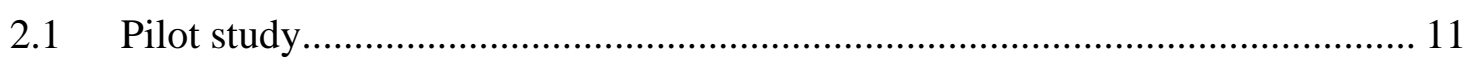

2.1.1 Observation and results of the pilot study.............................................. 12

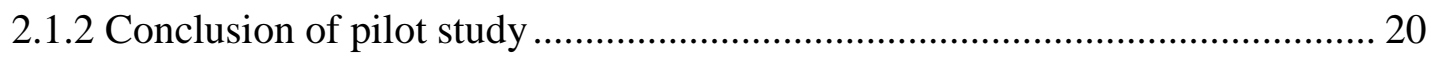

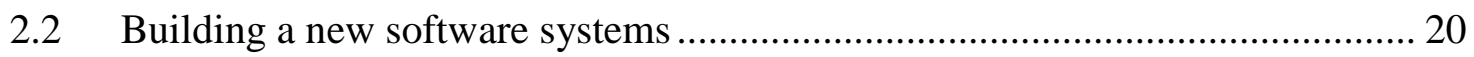

2.2.1 Development of the HCI feedback mechanism ......................................... 23

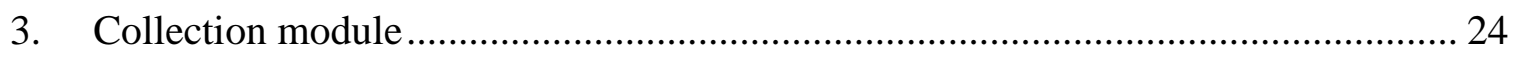

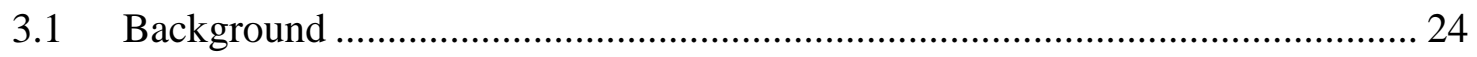

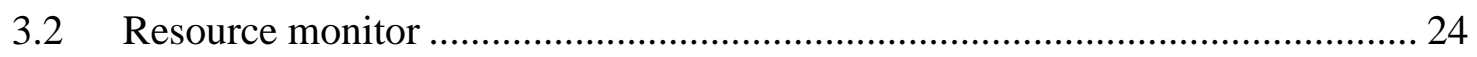

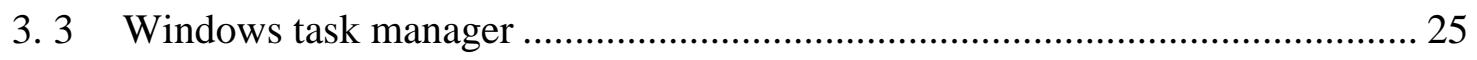

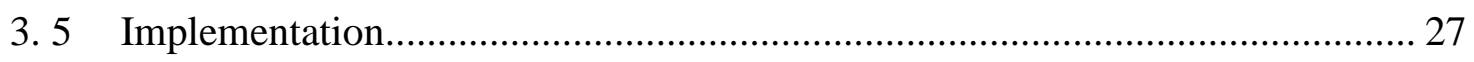

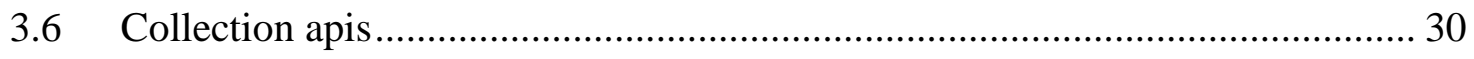

4. Transmission module ………………………………..................................... 32

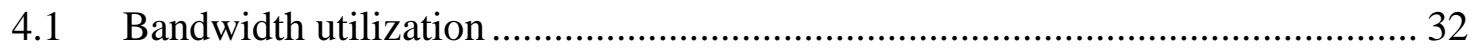

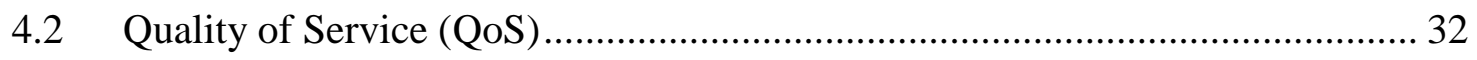

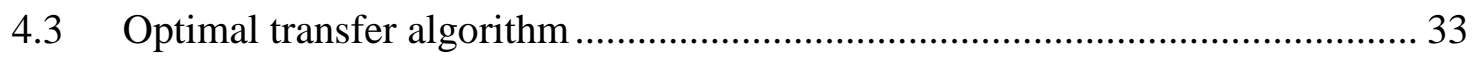

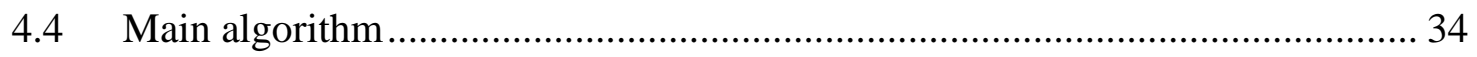

4. 5 TCP stream socket server application .......................................................... 38

4.6 TCP stream socket client application ......................................................... 43 


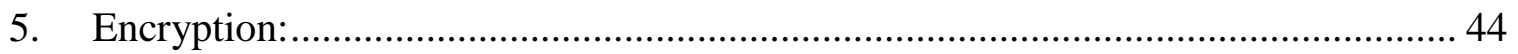

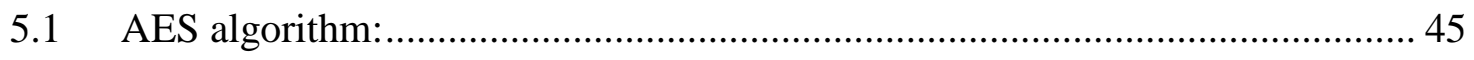

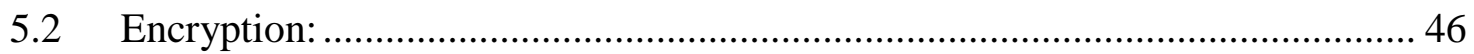

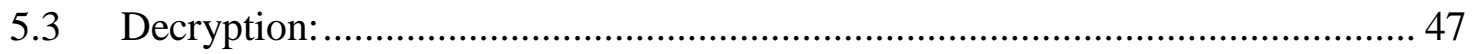

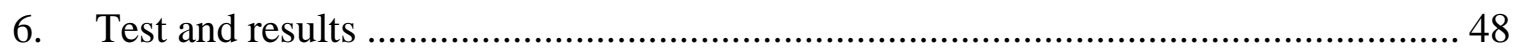

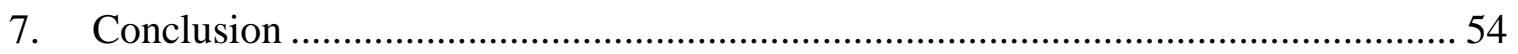

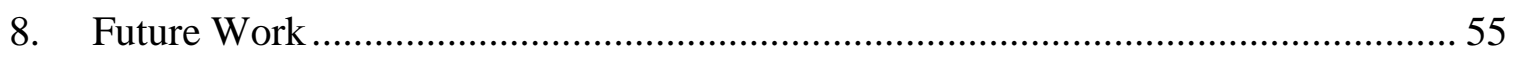

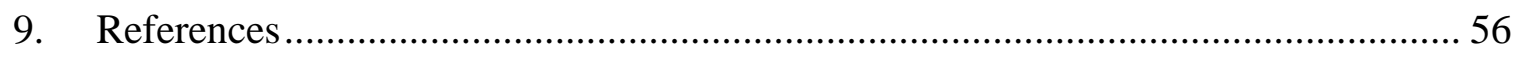




\section{List of Figures}

Figure 1: User monitor \& feedback mechanism for social scientific study on laptop

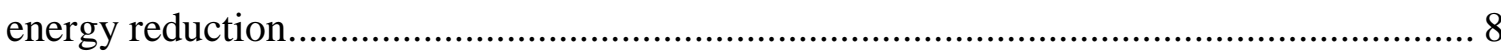

Figure 2: Computer operating state ......................................................................... 13

Figure 3: Samples collected by hour of day ................................................................. 14

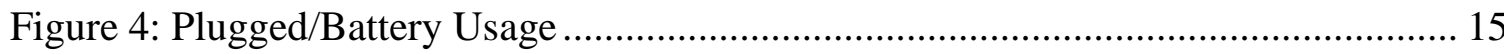

Figure 5: Top 9 processes by sample count ................................................................. 16

Figure 6: Top 10 processes by CPU consumption (seconds consumed during study

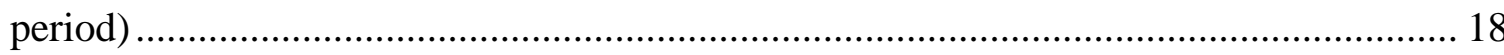

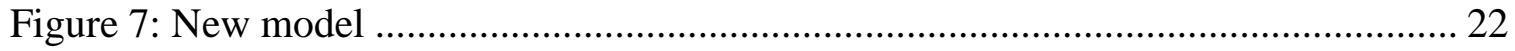

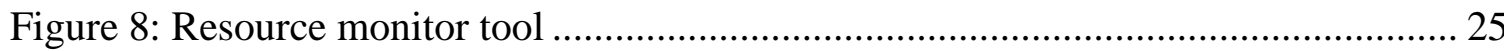

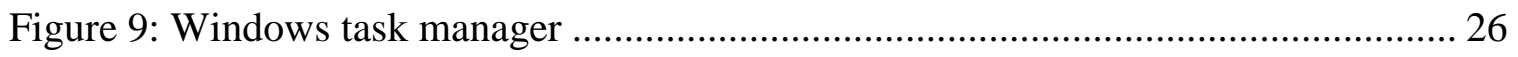

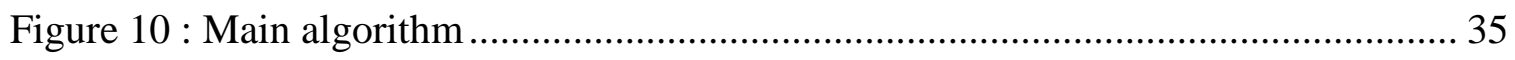

Figure 11: TCP client - server socket connection ......................................................... 40

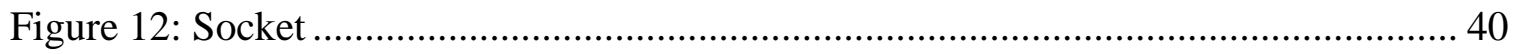

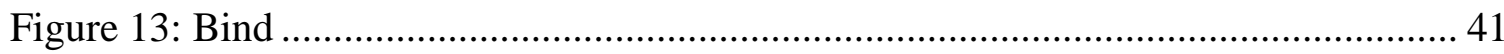

Figure 14: AES algorithm [17] ............................................................................... 45

Figure 15: The server is started and socket is opened for the client to send the data ....... 48

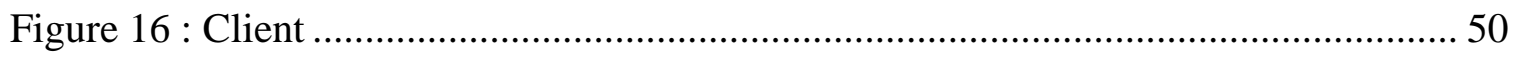

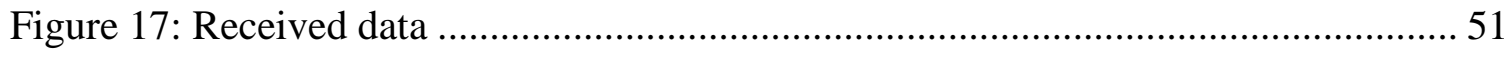

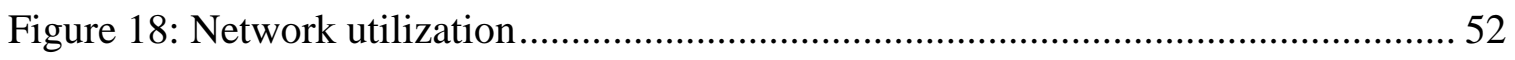

Figure 19: CPU Utilization .................................................................................. 53 


\section{List of Tables}

Table 1. Summary of data collection quantities..................................................... 13

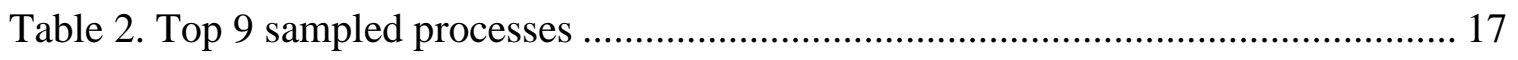

Table 3. Top 10 CPU consuming processes by category .......................................... 19 


\section{List of Code}

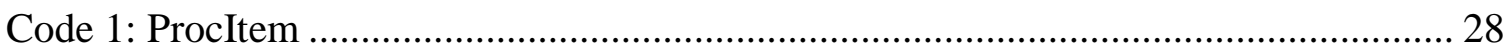

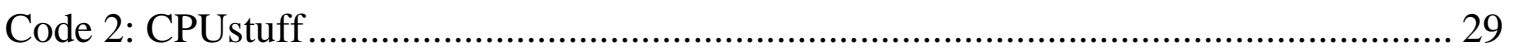

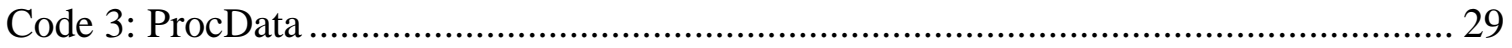

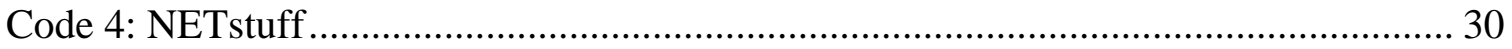

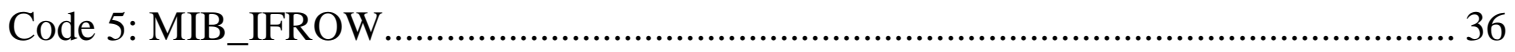

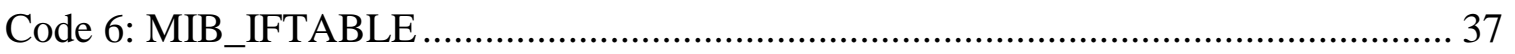

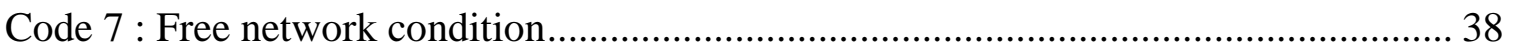

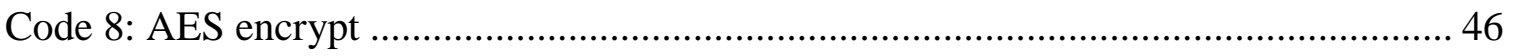

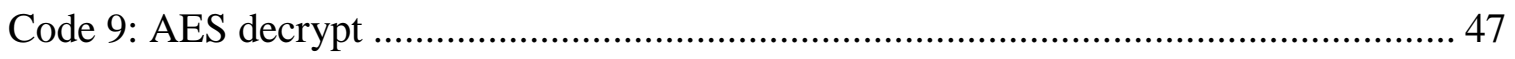




\section{Introduction}

Energy use related to electronic devices has skyrocketed, especially in the consumer electronics domain [1]. For example, the number of personal computers (PCs) around the world passed the one billion mark in 2008 [2]. The energy costs and related environmental impacts of office computers and networking equipment are well known [3][4][5], but residential and personal use of portable electronic devices also presents a growing concern.

Among all consumer electronic devices, laptop computers being portable and more energy efficient, now account for more than 50\% of PCs sold in America. The energy consumption levels of laptops vary depending on their laptop types and user operational modes [6]. Further, user behaviors play an important role in overall laptop energy consumption [7][8].

A research project was proposed by Dean of Engineering, Belle Wei, Prof. Morris Jones, and Prof Donald Hung, at San Jose State University called the "iGreen", to improve the efficiency of laptop energy usage by motivating users to change the way they use their laptops (behavioral change) for efficient energy consumption through persuasive technology. This proposed research will provide laptop designers with realistic feedback about consumer-usage and hence aid the design of improved laptop systems by investigating this area of research by tapping into the college student population, who are avid laptop users. A group of college students was selected because they are avid laptop 
users with $87.7 \%$ of them reporting laptop ownership [25], and user behaviors play an important role in the total laptop system power consumption [26].

In order to motivate user behavioral change, a study was performed to find how college students use their laptops, whether there are correlations between their usage and demographics (e.g., gender), their attitudes toward environmental issues, and to determine effective cognitive and social feedback mechanisms [26] to motivate more energy efficient behavior. The goal of the pilot phase is to explore and develop various components needed to build a laptop usage model, including the software tools for monitoring and collecting laptop usage data, processing and analyzing the collected data, and system-level laptop power measurement. It also helps to sharpen the questions asked of the participants on their attitude towards their relationship with the environment and technology. To obtain laptop system and usage data, a software monitor tool was developed and installed on the participants' laptop, and actual user data was collected.

This research was performed by monitoring the performance of laptop usage using a software monitor tool. The user behavior data was collected, which mainly consisted of information regarding computer resources like CPU, disk read/write, and network traffic.

The first version of the monitor tool collected some network information, which was unnecessary. This project is thus focused on redesigning the monitor tool to collect only the required information and to integrate a transmission module, which will send the collected data to the server for analysis when the network is free. 


\subsection{Objective of the project}

The objective of this project is to redesign the software monitor tool and add more features after the pilot study. This tool collects data from the user's system; constitutes information regarding computer resources like $\mathrm{CPU}$, disk read/write; automatically transmits the data to server for analysis purposes and to implements scientific study on laptop energy reduction by monitoring the user behavior and develop a feedback mechanism for the next experiment which is going to get conducted in Summer 2011 on 100 students. Figure 1 shows the block diagram for the main research proposed, named iGreen. This project is the part of iGreen that implements the collection and transmission module. This new model of the tool will be useful for the next experiment on 100 students to implement the feedback mechanisms for social and scientific study on laptop energy reduction.

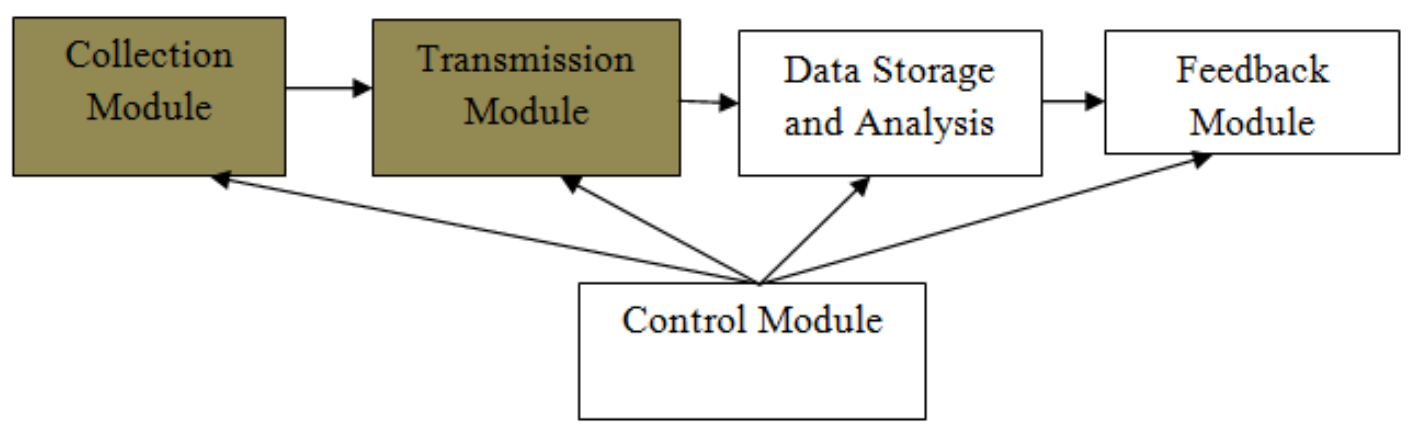

Figure 1: User monitor $\&$ feedback mechanism for social scientific study on laptop energy reduction 


\subsection{Outline of the project}

Section 2, begins with the pilot study which was conducted for the iGreen research, which is proposed to stimulate power consumption awareness and behavioral changes among laptop users via interactive energy-usage feedback with college students as the initial target group. Section 3 describes in detail about the background of collection module and its implementation for the monitor tool. In Section 4, the transmission module is covered, which is successfully deployed to transfer the data from client to server. Section 5, discusses AES encryption algorithm, which is implemented to provide the secure data transmission. In Section 6, the tests and results of monitor tool are discussed. 


\section{Background}

For the first time in the history of computing, laptop sales have exceeded desktop sales [6]. The dawn of the laptop era presents new challenges to the computing research community. Performance is no longer the be all end all of computing research because a new, significant requirement has cropped up: reducing the power consumption and improving the usage of battery rather, an important new goal has arisen, improving mobility. The key restriction to mobility is the constant need to be near a power outlet for recharging the laptop battery. Thus, extending battery life without compromising performance becomes a pressing concern.

To achieve this, the first step is to develop a baseline consumer laptop-usage workload, one different from current computer workloads that target the business environment, instead of consumer. Furthermore, exiting benchmarks, especially those in energy efficiency presume simple workloads, fail to reflect the complexity and variety of user behaviors. Examples are Energy Star[4], JouleSort[5], or BAPCo's MobileMark 2007 [6].

Under the research of iGreen project a pilot study was performed on 13 SJSU students. The goal of the pilot phase was to explore and develop various components needed to build a laptop usage workload; including the software tool for monitoring and collecting laptop usage data; processing and analyzing the collected data; and system-level laptop 
power measurement. It also helps to narrow down specific questions that can be asked to the participants on their attitudes towards their relationship with environment and technology.

\subsection{Pilot study}

A group of 13 SJSU student participants were recruited via a convenience snowball sampling procedure [34]. Survey data was collected from all 13 participants using the environmental attitudes inventory, a self-report measure of perceptions about the natural environment and policymaking. To obtain laptop system and usage data, a software monitor tool was developed and installed on the participant's laptops, and actual user data was collected for a period of four weeks.

The monitor tool, running in the background without any user interface, used sampling and event-driven mechanisms to gather data on user activities regarding local and web applications every seven to ten seconds. The information collected through the sampling process included the total CPU loading, displaying backlight levels, power source, battery conditions, network interfaces, network cumulative traffic in bytes and packets, as well as all user visible processes and their related resource consumption data. The latter included process creation time, execution time, disk read/write rates, system resources used, and the names of all .exe and .dll files that were loaded. Shutdown events and data on website file types retrieved were also collected

The monitor programs and result data were removed from participants' machines at the end of the study period. That data was transferred to a SQL data base for further analysis. 


\subsubsection{Observation and results of the pilot study}

The data was collected from 13 SJSU students for four weeks. That data was collected via sampling and event-driven mechanisms. The software monitor tool has created a repository with $3 \mathrm{~GB}$ of raw data (about 150-250 MB collected from each participant) containing real time laptop usage information. After performing the analysis, it was found that certain information for some known factors that significantly impact laptop power consumption was immediately extracted from the raw data.

Table 1 summarizes some of the information collected from the participants during the study period. The data collection and processing team knew the participants only by number.

\begin{tabular}{|l|l|}
\hline Amount & Item \\
\hline $1,067,539$ & Time point samples \\
\hline $10,792,762$ & Process sample points \\
\hline $2,862,556$ & CPU seconds tracked to Processes \\
\hline 128,986 & Process to DLL bindings \\
\hline 832 & Unique processes \\
\hline 461,572 & Input intervals \\
\hline $5,122,169$ & Network interface items \\
\hline
\end{tabular}




\begin{tabular}{|l|l|}
\hline 385 & Shutdown events \\
\hline 3 & Operating system releases \\
\hline
\end{tabular}

Table 1. Summary of data collection quantities

Figure 2 shows the aggregated time distribution for different laptop operation states in terms of shut (off), sleep, and active.

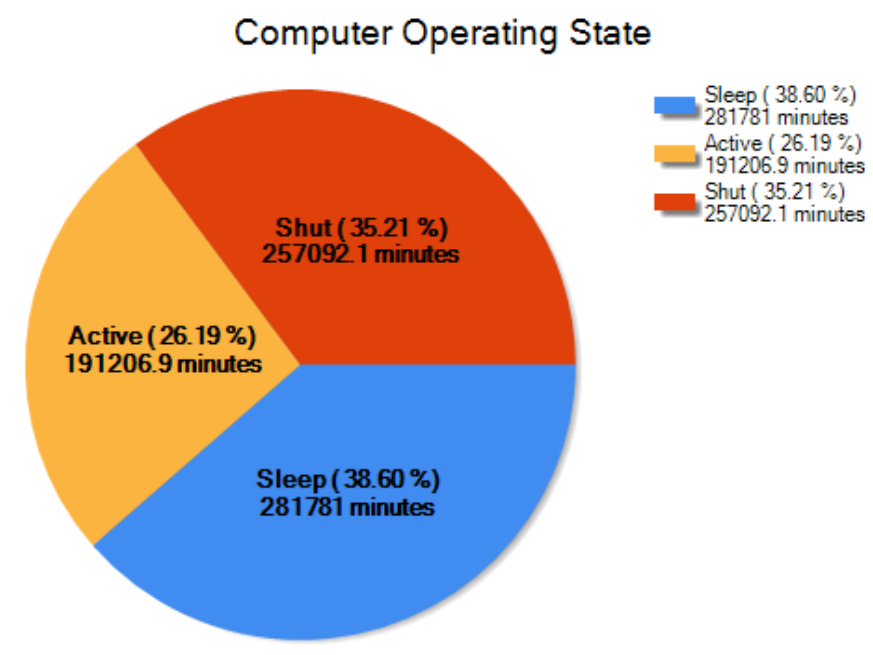

Figure 2: Computer operating state

Figure 3 shows the number of samples collected by time of day during the study period. Samples were collected whenever the machine was running. This was used as an indicator of the amount of time the user has the machine active or idle. 


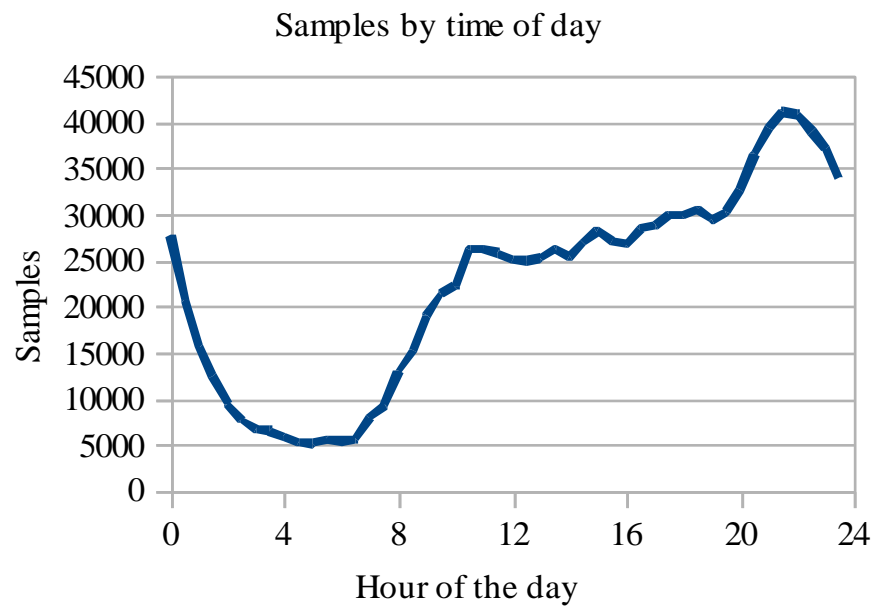

Figure 3: Samples collected by hour of day

It was found that the peak numbers of machines were powered on between 8PM and midnight. The data showed limited student use in the morning. Several of the participants in the study never turned the notebook off. The notebooks ran with the lid closed and they kept charging throughout the day. There were 385 shutdown events recorded in the study. This was about once per day for each study participant.

\section{Battery power}

The pilot study results included information about AC/Battery mode, the operation state (off, sleep, idle or active), and brightness levels of backlight displays. It was found that the participants' laptops were running on average $27.8 \%$ of the day (6.5 hours/day). While running, they are plugged to the AC power outlet on average $83 \%$ of the time [Figure 4]. All of the pilot-study participants' laptops ran with a high-performance, highpower profile while on AC mode.

The energy-consumption difference between $\mathrm{AC}$ and battery modes for a laptop running the same application could be significant. For instance, our laboratory power 
measurement showed it to be $26 \%$ (higher for AC) for running a 40-minute DVD video. This difference is attributed to the setting of the laptop's power management profile to low power while on battery. The higher power consumption for the laptop on AC coupled with $83 \%$ AC mode operation in the pilot group suggests a fruitful area for energy savings for both users and designers.

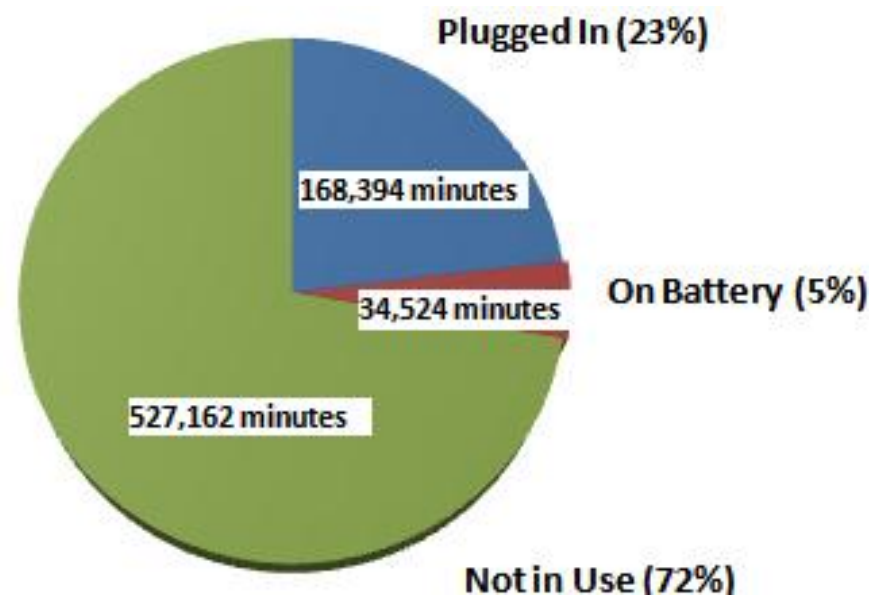

Figure 4: Plugged/Battery Usage

For users, this means there is the possibility of setting the laptop's power management profile to low power, even though it is on AC or switching its mode from AC to battery. For designers, this means there is the possibility for a lower power design for the laptop on AC mode, even though the battery life is not a design concern for this mode. Pilot data showed that, while on $\mathrm{AC}$, the automatic sleep or hibernate mechanism was typically disabled in the participants' laptops. Convincing users to change their laptop operation states (sleep/hibernate/shutdown) while on AC can result in significant energy savings [33]. 


\section{Processes in use}

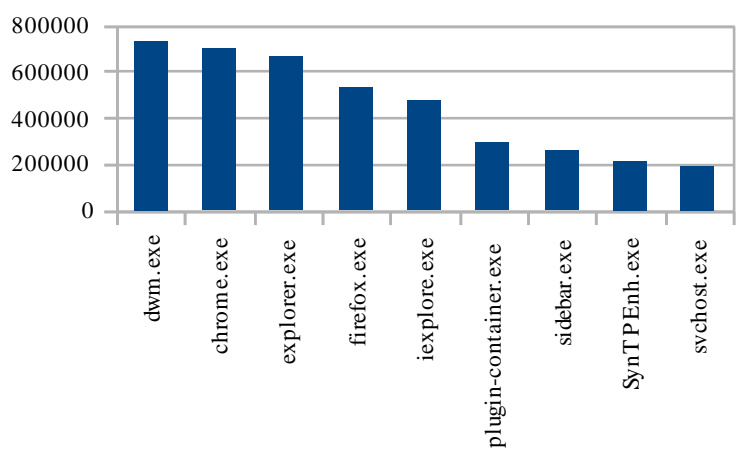

Figure 5: Top 9 processes by sample count

The system collected information on user visible processes per each sample. On average, there were 10 visible processes used by the participant's machine per each sample. Five of the top 10 user visible processes are related to operating system functions. The frequency of sighting is shown in figure 6 for the top 9 sighted processes. Table 2 identifies user application processes.

\begin{tabular}{|l||l|l|}
\hline Name & User/OS & Description \\
\hline Dwm.exe & OS & Display manager \\
\hline Chrome.exe & User & Web browser \\
\hline Explorer.exe & OS & User shell \\
\hline Firefox.exe & User & Web browser \\
\hline Iexplore.exe & User & Web browser \\
\hline $\begin{array}{l}\text { Plugin- } \\
\text { Container.exe }\end{array}$ & User & $\begin{array}{l}\text { Second process for Firefox. } \\
\text { Runs flash typically) }\end{array}$ \\
\hline Sidebar.exe & OS & User shell application \\
\hline
\end{tabular}




\begin{tabular}{|l|l|l|}
\hline SynTPEnh.exe & OS & Notebook touch pad interface \\
\hline Svchost.exe & OS & Dynamic linking support \\
\hline
\end{tabular}

Table 2. Top 9 sampled processes

These numbers do not show the amount of resources consumed, just the amount of time the application process was present. The sum of the three web browser samples was about 2.5 times greater than the number of user shells. This implies that at any one time, a typical user had 2.5 browser applications open. Each browser application had multiple tabs or sessions active within the process.

The system collected the CPU times for each process and sample. Cumulative values were recorded, and the resources consumed in each sample period were computed by the differences from a previous period. Figure 7 shows the top 10 processes by user CPU consumption. 


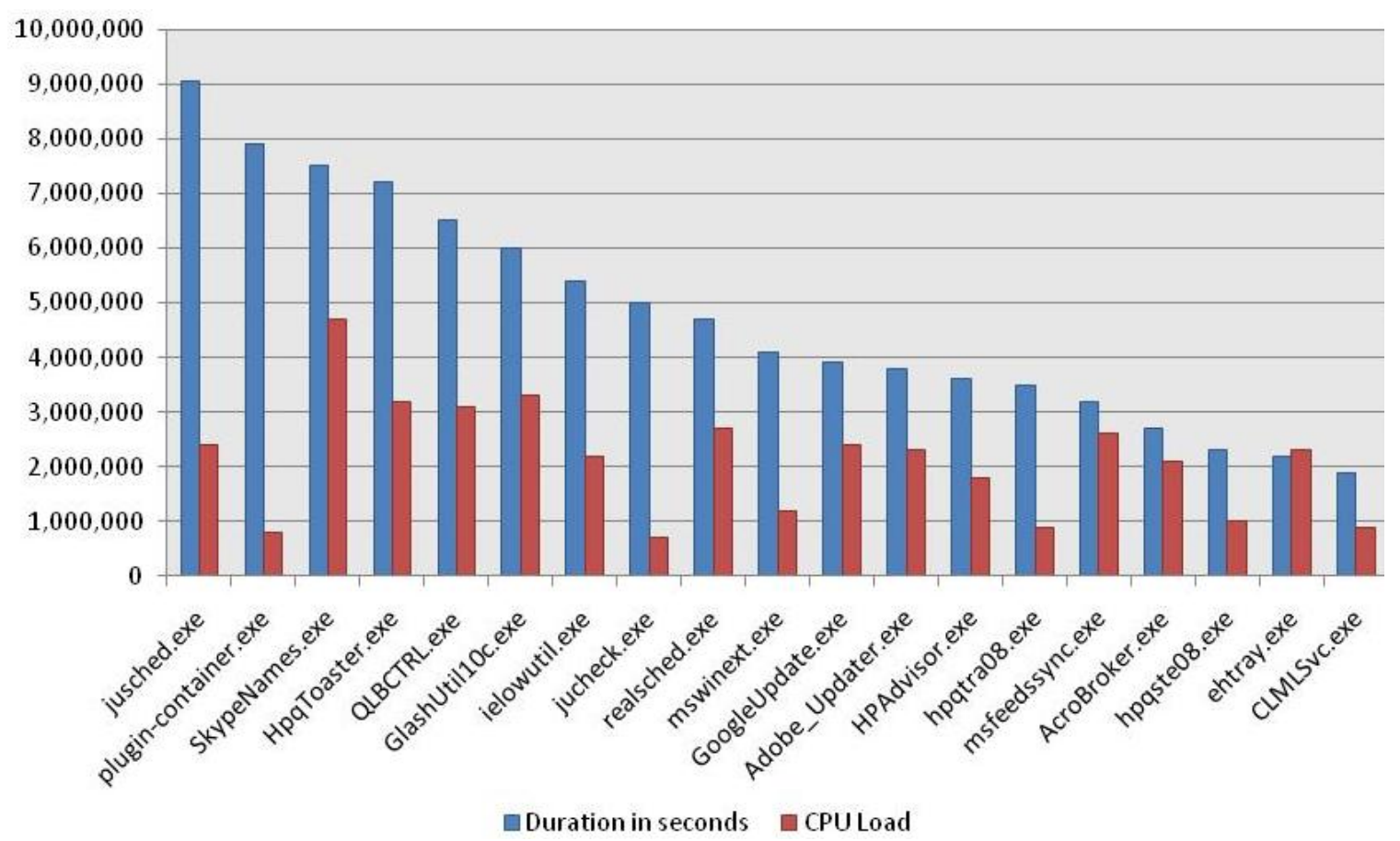

Figure 6: Top 10 processes by CPU consumption (seconds consumed during study period)

The applications were analyzed and placed in a set of broad categories. This is shown in table 3. The system reported both user and kernel times allocated to the application processes. It was observed that some applications had more kernel time than user time. There were 2.2M CPU seconds of user times reported, and $608 \mathrm{~K}$ seconds of kernel times. This is an area for further study.

\begin{tabular}{|c|l|}
\hline \hline Category & Total user CPU seconds \\
\hline Browsers & $1,217,000$ \\
$\bullet \quad$ firefox.exe & \\
$\bullet \quad$ chrome.exe & \\
$\bullet \quad$ iexplore.exe & \\
\hline Media & 373,000 \\
$\bullet \quad$ convertXtoDVD.exe & \\
\hline
\end{tabular}




\begin{tabular}{|c|l|}
\hline$\bullet \quad$ wmplayer.exe & \\
\hline $\begin{array}{c}\text { Games } \\
\bullet \quad \text { java.exe }\end{array}$ & 97,000 \\
$\bullet \quad$ AttackonPearlHarbor.exe & \\
\hline Operating System & 89,000 \\
\hline
\end{tabular}

Table 3. Top $10 \mathrm{CPU}$ consuming processes by category

Browsers consumed over half of the total CPU times of the study. Outside of these groupings, all of the other applications consumed approximately $25 \%$ of the CPU resources during the study period. The notebooks appeared appear to be used primarily for web type applications. Word processing is number 17 in the list of CPU consumption, and just beat instant messenger. Total word processing consumed 28,898 seconds CPU time. One participant consumed more CPU time playing a game than the entire study group consumed during word processing. Students may justify portable computers for educational purposes, but they appear to use them primarily for browsing, media applications and games.

Background applications are considered to be the main area of research. Indeed, the application that used most CPU resources in the pilot study was a background application. As our pilot study demonstrated, a significant amount of CPU resources were consumed by background applications, which include messengers, application updaters, VoIP (Voice over IP) listeners, and screen effects such as information feeds and photo albums that constantly change [Figure 4]. These applications tend to be installed with software packages; the user seldom installs them directly. Behavioral changes could include changing the auto start settings of these applications. 


\subsubsection{Conclusion of pilot study}

The pilot study for the iGreen project was completed. As the project iGreen continued the recorded usage data was aggregated and represented by a set of attributes. Each represented a certain type of activity with its energy consumption information obtained from laboratory measurement. Since the recorded usage data was time stamped, the different usage patterns (e.g., time spent on a particular type of activity, with the usage intensity indicated by numbers of overlapped attributes in the same time interval) of each user were extracted and presented in the form of pattern vectors. Furthermore, statistical pattern classification techniques were applied on a large set of user samples to form a patterned space. This patterned space consisted of various classes of usage patterns with known energy consumption levels, and to developed a pattern classification mechanisms [28],[29]. Based on which, the energy-use feedback can be provided to the user.

A new study of a group of over 100 students is scheduled for this summer using a newly developed software system with a transmission module which automatically transfers client data to server. After performing data analysis on the server, the user feedback is provided for the purpose of reducing laptop energy.

\subsection{Building a new software systems}

In preparation for the next experimental phase, software systems will be developed to implement the feedback mechanisms designed by the behavioral science team. They will 
monitor user behaviors, track behavioral changes, as well as the infrastructure, to ensure the privacy and security of user data that will be transmitted and stored.

In new collection process, the participants' data will be transported to a server over a public network using three layers of encryption. They will data will first be encrypted using the public key encryption of AES cryptography. This public key system provides user protection, and ensures proper participant identification. This is done using openssl libraries. The data will be decrypted on the server and placed in SQL (Structured Query Language) data base for analysis.

The new plan is proposed to have two servers, one secure primary server and a secondary server. The primary server will process data for general dissemination from the raw data and place it them on the secondary server. The secondary server will have no participant identifiable data. 


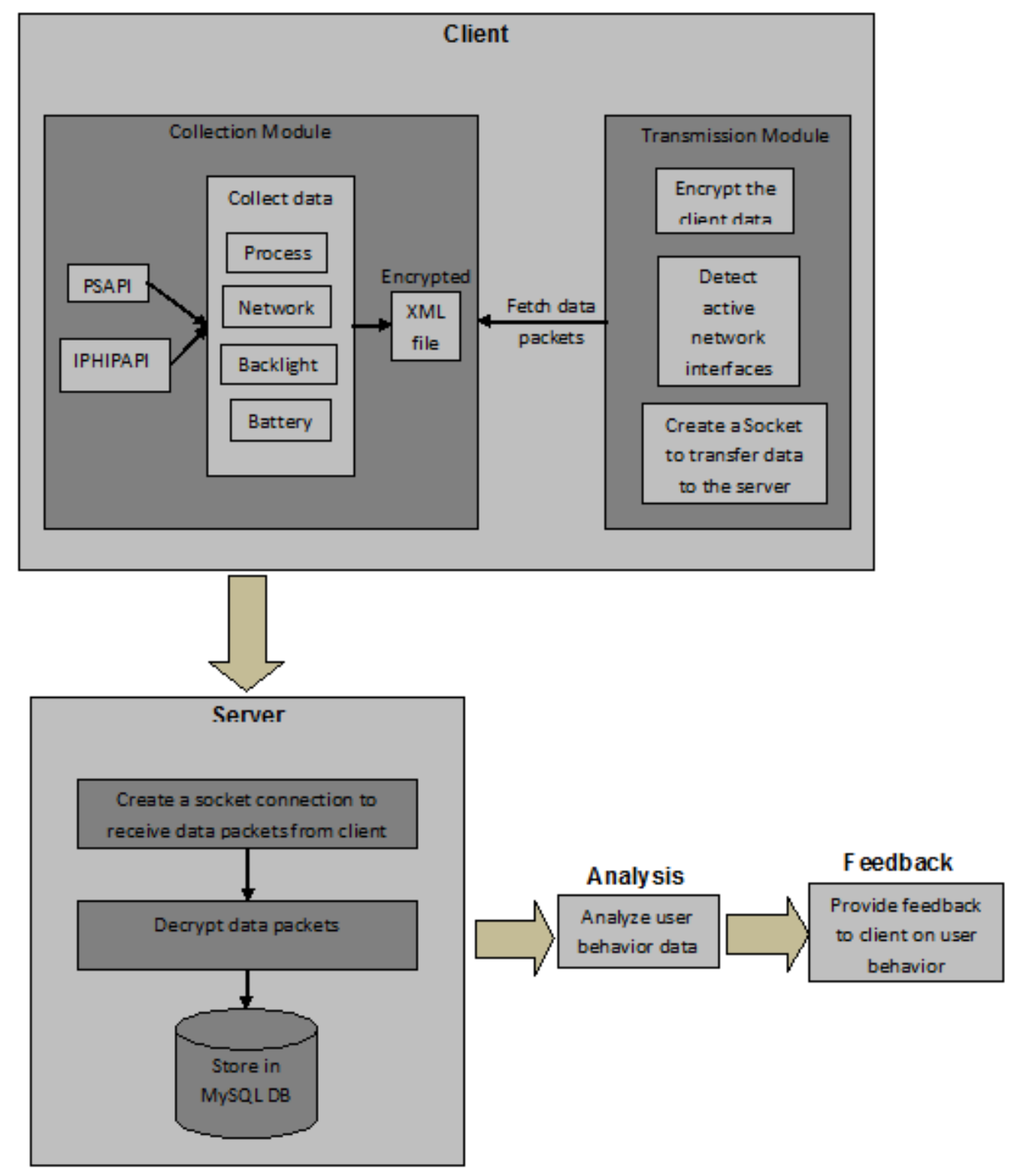

Figure 7: New model 


\subsubsection{Development of the $\mathrm{HCl}$ feedback mechanism}

Working with the engineering team, the behavioral science team will develop an energyuse feedback mechanism with a compelling user interface design which will effectively educate and motivate users to improve their laptop use for better energy efficiency. The feedback mechanism monitors the state of targeted energy conservation acts and initiates a trigger on the screen of a laptop, once the state exceeds a threshold energy profile. While we might expect the participants to be self motivated to respond to the trigger to some extent, our interface design informed by the HCI (human-computer interaction) literature will incorporate visualization [10], feedback [11][12][13], discovery and motivation design techniques [14], and easy actionable steps [15][16] to make a significant difference (i.e., to strengthen motivation and simplify a needed ability enough for laptop users to take targeted action more successfully).

Our objective is to design a dynamic and compelling visual user interface that motivates participants to take actions, implicitly educating them towards adopting a more selfdirected energy saving behavior in the long run. When a user further interacts with the interface, s/he will discover more details about the specific user-controlled behaviors. The machine will state the underlying the energy use indicator, as well as motivating patterns and trends (perhaps with competitive elements). Once the user responds to a specific "trigger" by taking the corresponding action, its underlying energy profile will change to a lower state and the trigger will then disappear from the laptop screen. 


\section{Collection module}

The collection module is a component of the monitor tool that assembles an array of statistics to measure the user's behavior.

\subsection{Background}

A Microsoft tool known as the Resource Monitor performs a similar function like monitor tool.

\subsection{Resource monitor}

The Windows Resource Monitor is a tool for understanding how your system resources are used by processes and services. In addition to monitoring resource usage in real time,

the Resource Monitor can help you analyze unresponsive processes; identify which applications are using files, and control processes and services. [22] It gathers information regarding CPU, Memory, Disk, and Network. 


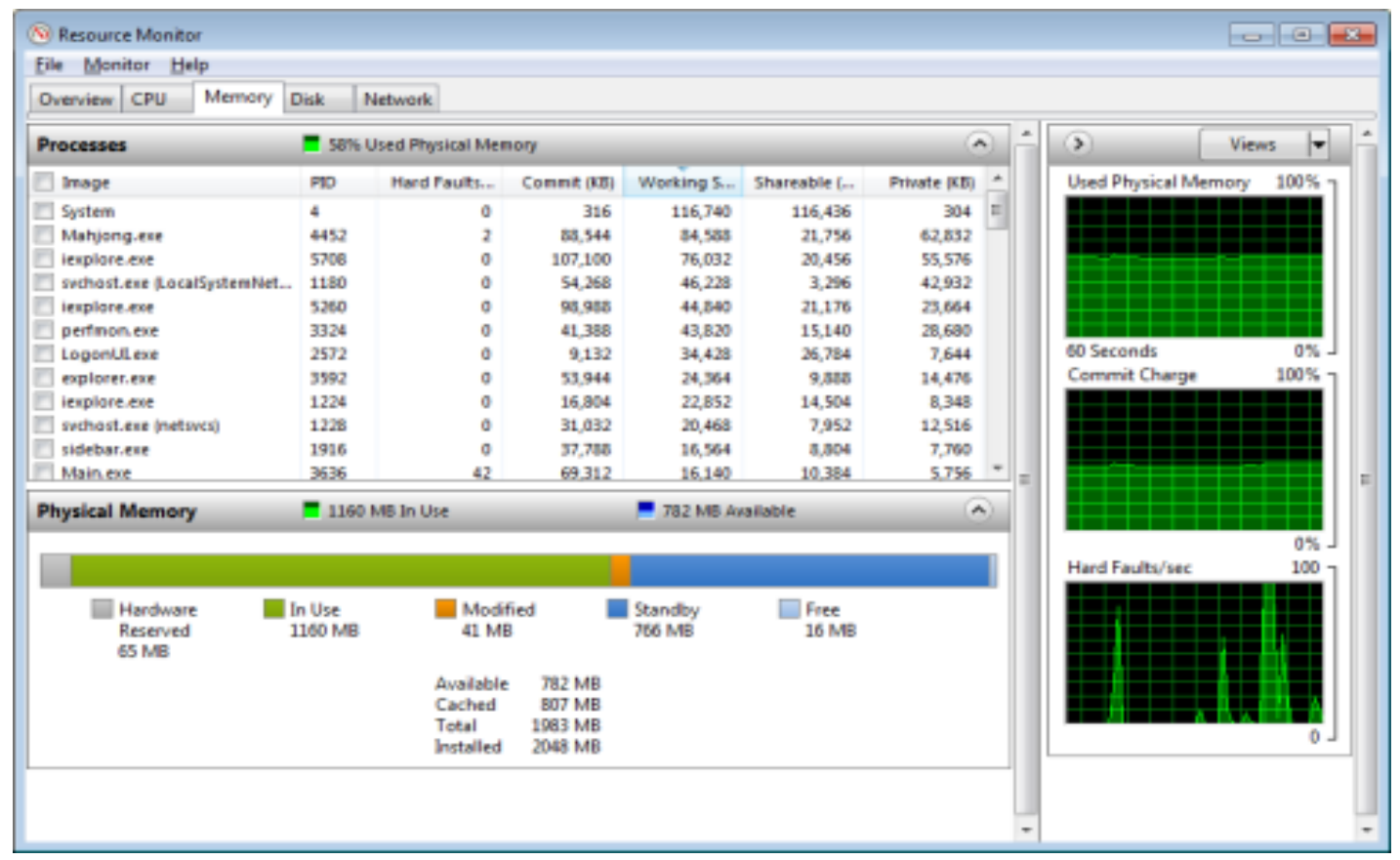

Figure 8: Resource monitor tool

\section{3 Windows task manager}

Windows has another tool known as task manager, which is available in all versions of windows starting from Windows NT. It provides detailed information regarding the computer performance like CPU usage, commit charge and memory information, network activity and statistics, logged-in users, and system services. It has the ability to set priorities by a processor affinity along with the functionality of forcibly terminating the processes and shut down, hibernate and log off from windows [24]. 


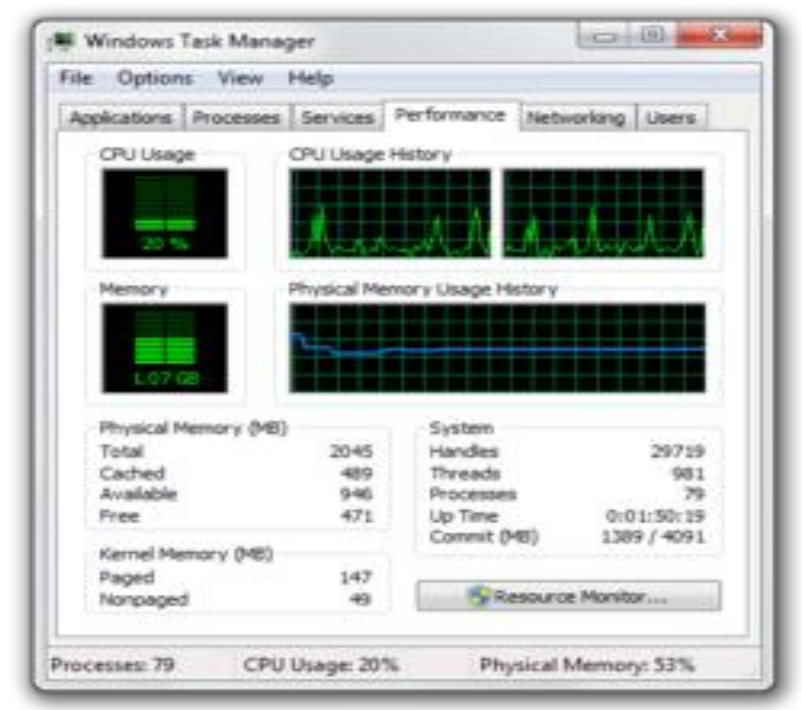

Figure 9: Windows task manager

There are a few reasons why we needed to develop the monitor tool even though there are other tools with a similar functionality, like resource monitor and task manager, which are a utility in windows:

- The Resource monitor utility for Windows is only present in Windows 7 and Vista, while it is not present in Windows XP.

- The Resource monitor tool and task manager cannot log the data, which can be further analyzed to check the behavior of the user.

- The Resource monitor is developed to provide feedback to the user regarding his/her behavior and to reduce power waste- a functionality which is not available in any of the tools like resource monitor and task manager. 


\section{5 Implementation}

To obtain a laptop system and usage data, a software monitor tool was been developed and installed on the participants' laptops. The actual user data was been collected for a period of four weeks during the pilot study. The monitor software, running in the background without any user interface, used two mechanisms for data collection: sampling and event driven. The sampling mechanism initiated a collection process that gathered information about user and system activities following a 5-second pause. The collection process took two to five seconds to complete due to system scheduling of operating system calls. The sampling period was then about seven to ten seconds. The information that was collected in this way included the total CPU loading, displayed backlight levels, power source, battery conditions, network interfaces, network cumulative traffic in bytes and packets, as well as all user visible processes and their related information. The process related information included creation time, execution time, system resources used, and the names of all .exe and .dll files loaded. Shutdown events are also collected. Data collection based on event driven mechanism was activated by a network request with a destination of port 80 and "GET" at the beginning of the message. It then monitored web-based traffic requests, and collects only the file type information. This data, together with the network cumulative traffic data, provided information on the web traffic flow.

The following code sections give information about the variety of data collected using the collection routine of this monitor tool. 


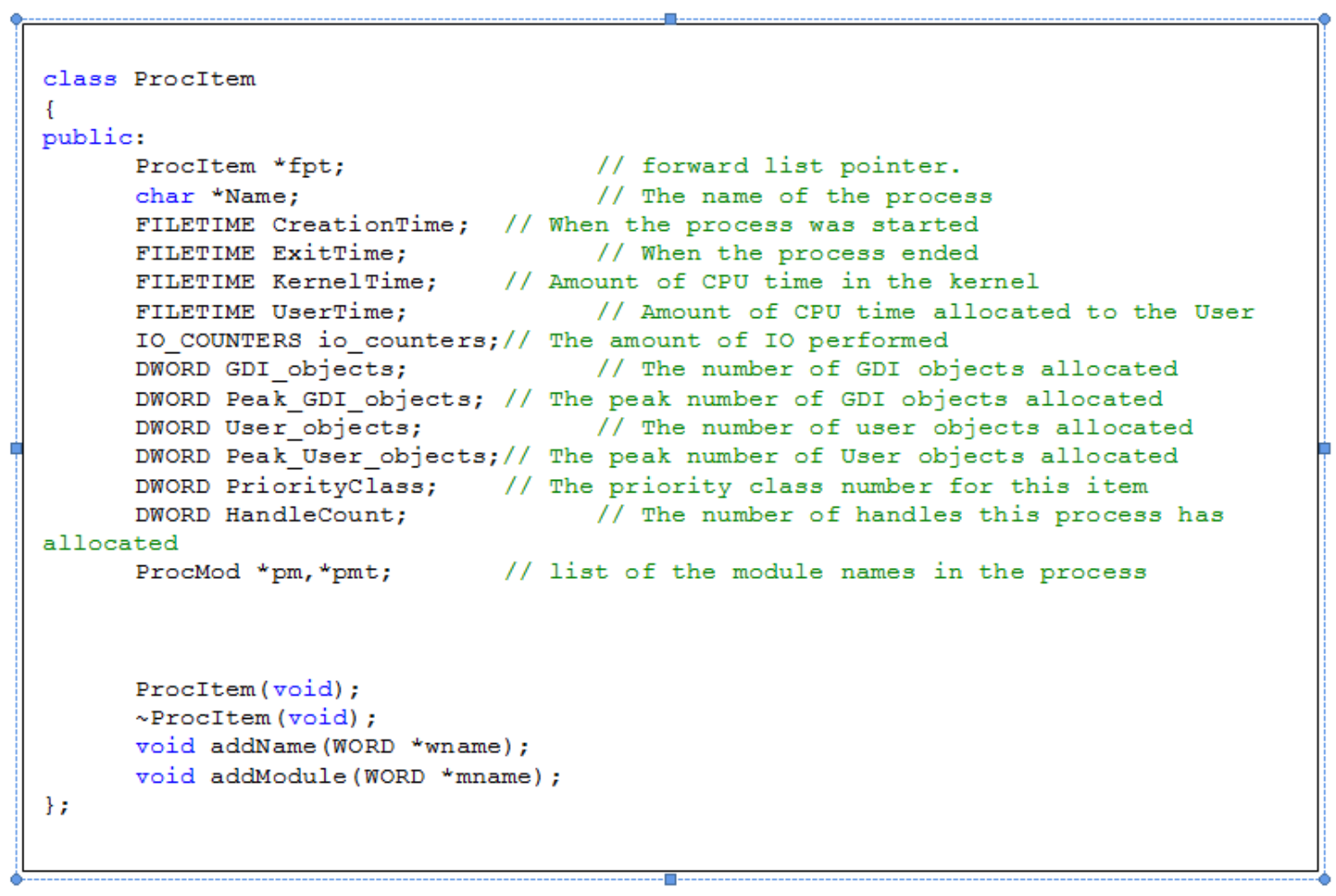

Code 1: ProcItem 


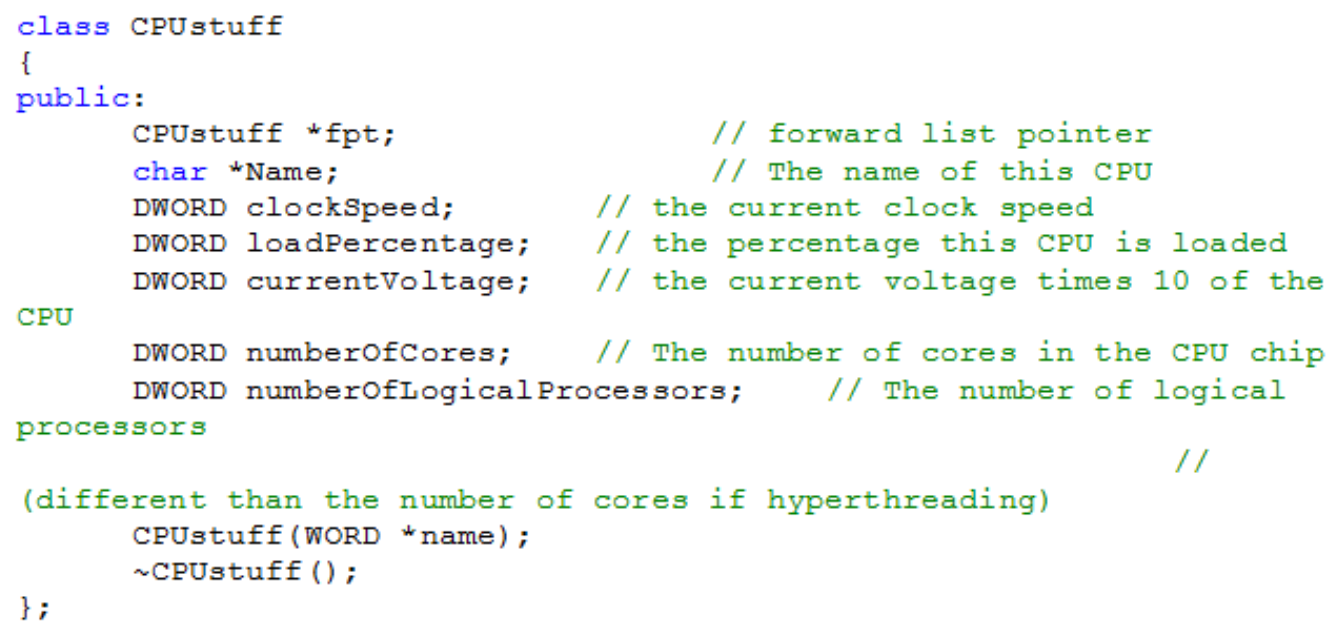

Code 2: CPUstuff

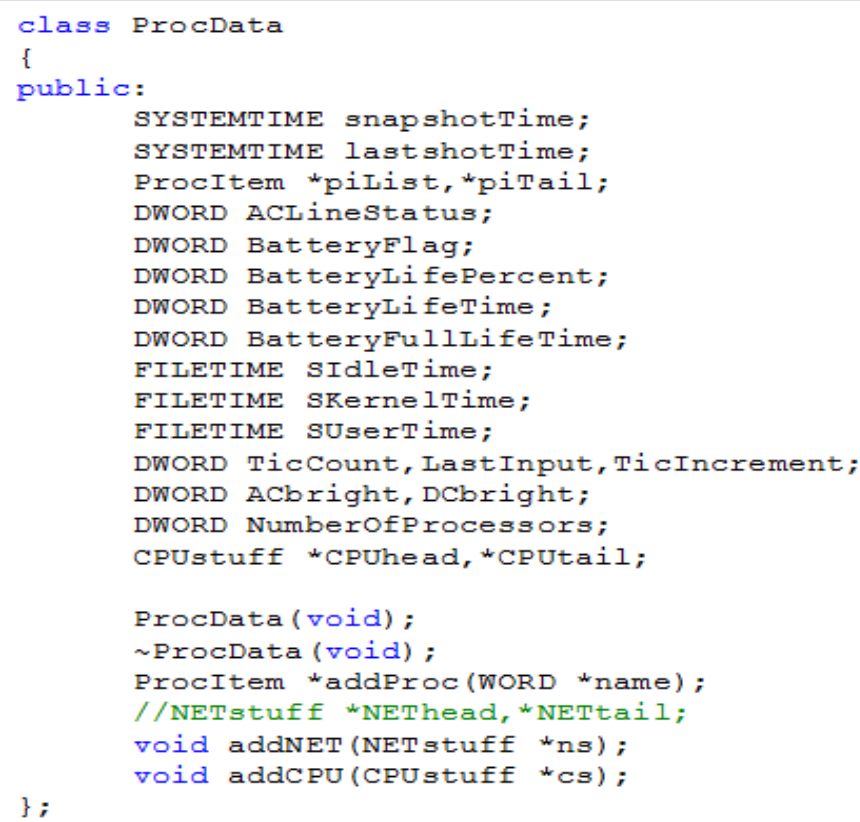

\section{Code 3: ProcData}




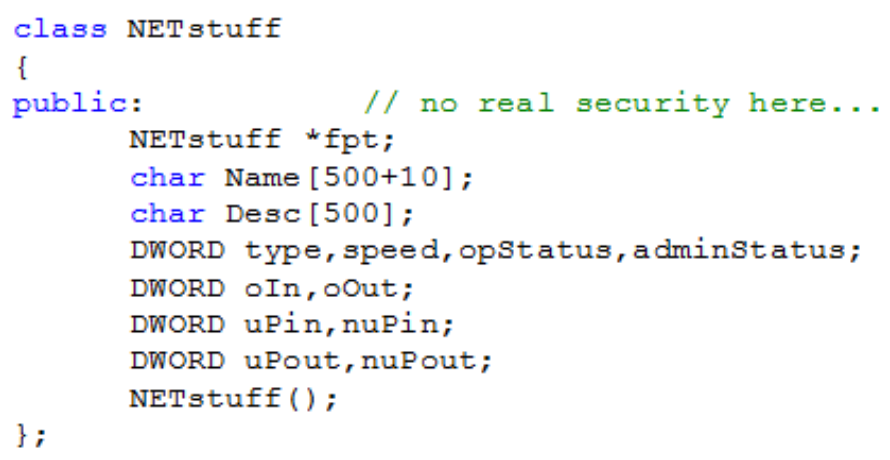

Code 4: NETstuff

The above code sections reflect various types of data collected using different Apis. This Apis can be summarized as follows:

\subsection{Collection apis}

\section{Iphlpapi.h}

This header file is implemented in the msdn library. It provides functionality to collect the information related to network interface; such as the name of network interface, type of network interface, network speed, operational status, unicast packets in \& out and non -unicast packets in \& out. [31]

\section{Psapi.h}

The process status application programming interface (PSAPI) is a helper api implemented in the msdn library- which gives process information like: device drivers 
process creation time, ending time, user time, kernel time, its GDI object details, handle count and process module details.

The battery information like AC indicator flag, battery flag, and battery life time is obtained from the power status class in "Windows.Forms" namespace.

The System information like system idle time, kernel time, tick interval, last input time, display $\mathrm{AC} \& \mathrm{DC}$ brightness along with $\mathrm{CPU}$ information like number of cores and number of processors is also gathered using the monitor tool [30]. 


\section{Transmission module}

Transmission module is a component implemented in the monitor tool which acquires the data from the collection module deployed on the client side and sends it over to database on server side.

\subsection{Bandwidth utilization}

Bandwidth is the primary measure of computer network speed. It represents the capacity of the connection. The greater the bandwidth the better the network performs. Bandwidth is the amount of data that passes through a network connection over time as measured in bps. It refers to the data rate supported by a network connection or interface. It is not the only factor that contributes to the network speed. Another key element for the network performance is latency. The latency refers to the several kinds of delays typically incurred while processing the network data [18].

\subsection{Quality of Service (QoS)}

Quality of service is the ability to give priorities to different applications and users. It is to guarantee a certain level of performance to a data flow. Hence, a required amount of bit rate, delay, jitter, packet dropping probability and/or bit error rate may be guaranteed. QoS also guarantees network capacity for real time streaming multimedia 
applications that require fixed bit rate and are delay sensitive, and for cellular data communication.

The network protocol, which supports QoS provides an opportunity for traffic contract with the software application by reserving capacity in the network for each node during the session establishment phase. It may monitor the performance during the session and calculate the data rate. It may also delay the performance by dynamically controlling and scheduling the priorities in the network nodes. Thus, QoS may release the reserved capacity in the tear down phase.

QoS cannot be achieved by the best-effort network service, however the alternative to complex QoS is high quality communication over a best effort network by over provisioning the capacity to achieve sufficient peak traffic load. Thus, by over provisioning the capacity, we can get rid of network congestion and therefore eliminate the need of QoS mechanism [21].

\subsection{Optimal transfer algorithm}

The optimal algorithm provides following facility,

- To fully utilize the communication bandwidth,

- To preset the bandwidth consumption on a needed level,

- And helps to deal with the situation, where any client unnecessarily requests too high of an update rate [20]. 
There are possibilities of two types of disadvantages in the client-server connection when the application reads or writes the data in different process variables. They can be summarized as follows:

1. Clients need to communicate according to the priority of the process.

2. Different clients send the data to the server at a time in whole communication path.

To reduce communication overhead, real time process data needs to be read in blocks. These blocks can be grouped. Each group can be assigned a sampling rate and can be scheduled in the sampling queue as one item. This sampling rate is a function of client requests and limitations set in the server configuration. The scheduling algorithm minimizes the difference between the real time sampling rates to the evaluated sampling rate [20].

There are few factors to consider while the data transfers on the network like: transfer scheduling, resource reservation for guaranteed-bandwidth, advance reservation and time constraints.

After considering the above factors, an algorithm was implemented to transfer the data collected using the collection module of the monitor tool, from client to server when the client network is free.

\subsection{Main algorithm}




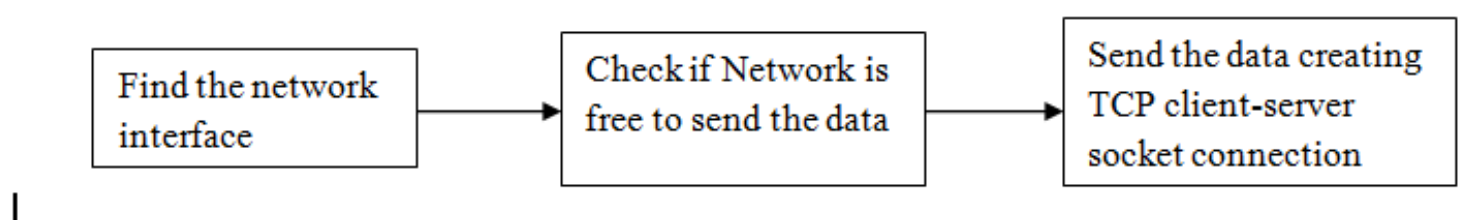

Figure 10 : Main algorithm

\section{1) To find the network interface:}

The network interface details are obtained from the two major functions MIB_IFROW and MIB_IFTABLE present in the msdn library like winsock2.h and iphlpapi.h to find the details about the network interface.

The following structure of MIB_IFROW is used to find the detailed information about particular interface

- The MIB_IFROW structure stores information about a particular interface. 


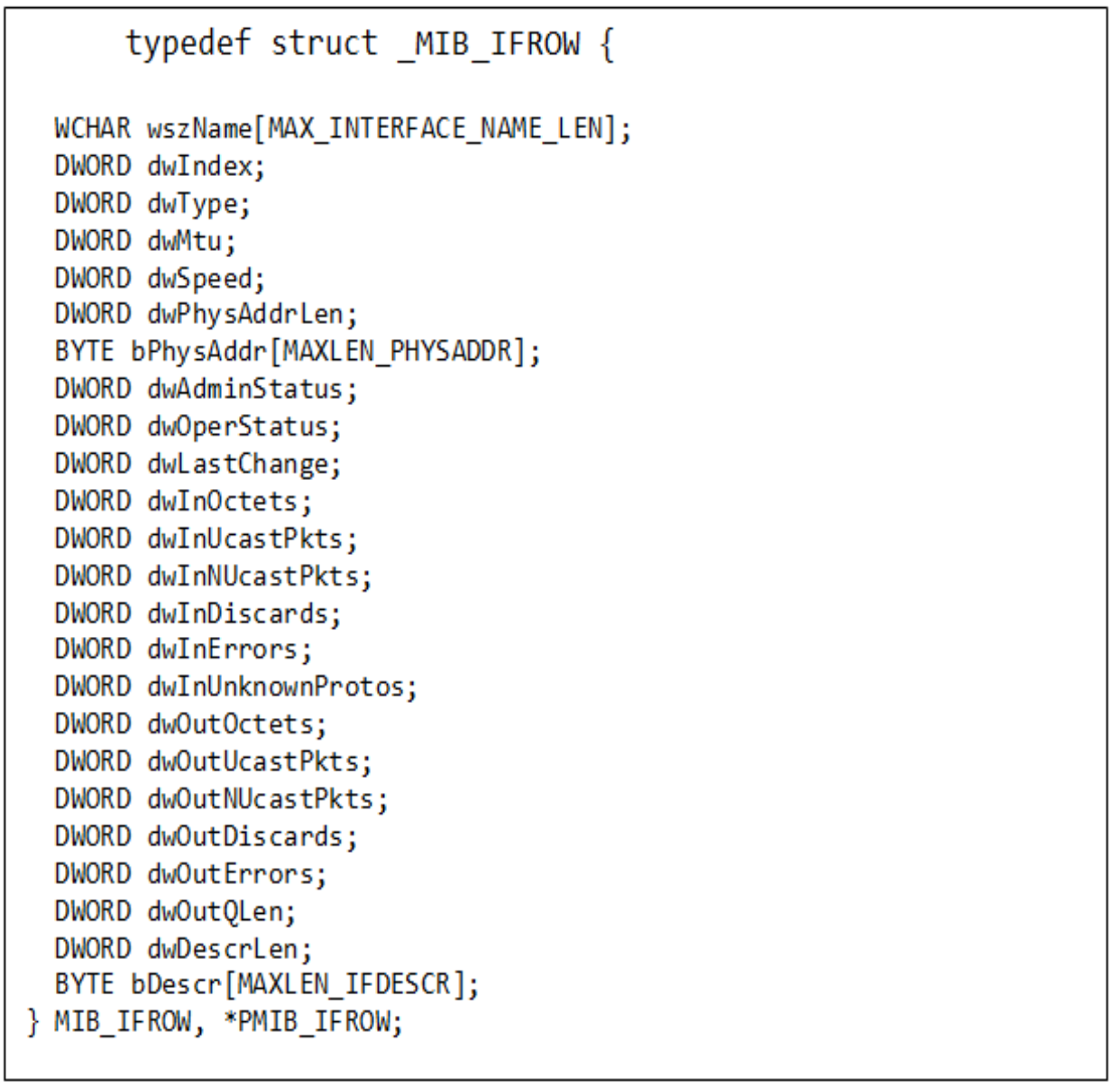

Code 5: MIB_IFROW 
- The MIB_IFTABLE structure contains a table of interface entries, which were connected and used by the user.

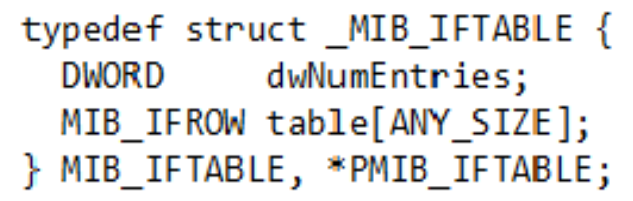

Code 6: MIB_IFTABLE

\section{2) Check if Network is free to send the data:}

The network data can be transferred from the client to the server when the client side network is free. To check the free network, the IN \& OUT discards and IN \& OUT errors are considered.

In Errors \& Out Errors

Type: DWORD

The number of incoming and outgoing packets that were discarded because of errors.

In Discards \& Out Discards

Type: DWORD 
The number of incoming and outgoing packets that were discarded even though they did not have errors.

The above two factors will help to find if the client network is free enough to send the data to the server. So, if following condition satisfies, then we can consider that the client network is available for sending the collected data from the collection module of the monitor tool.

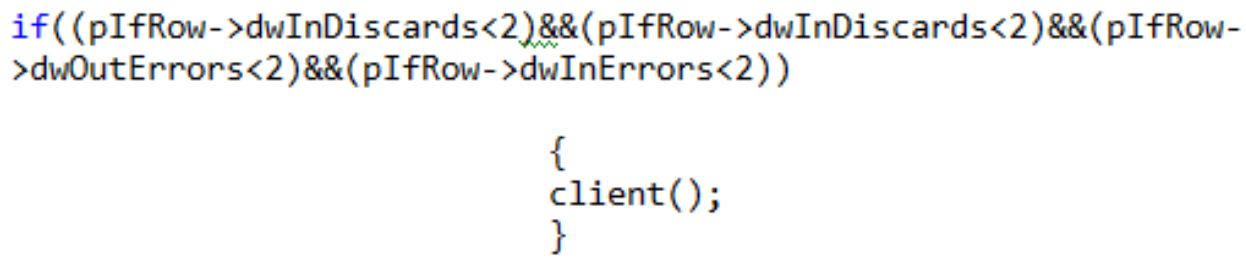

Code 7 : Free network condition

In \& out discards along with In \& Out errors are less than 2.

3.) Create TCP socket connection described in the next section.

\section{5 TCP stream socket server application}

The sockets are a protocol to create a connection between the processes. It can create either a connection based or connectionless connection. The main socket characteristic is AF_INET, which provides the format of a host and port number. The connection based socket communicates between client and server, wherein the server waits for a connection from the client. 
The main api's used for establishing the connection are as follows:

- Socket: To create a socket of particular type.

- Bind: It allocates a name to the socket.

- Listen: It specifies the number of pending connections that can be queued for a server socket.

- Accept: server accepts a connection request from a client.

- Connect: This API is to create a client request for connection with server.

- Send: Write to connection

- Recv: Read from connection

- Shutdown: End of the call

The following block diagram helps to understand the steps to establish the client server connection: [23] 


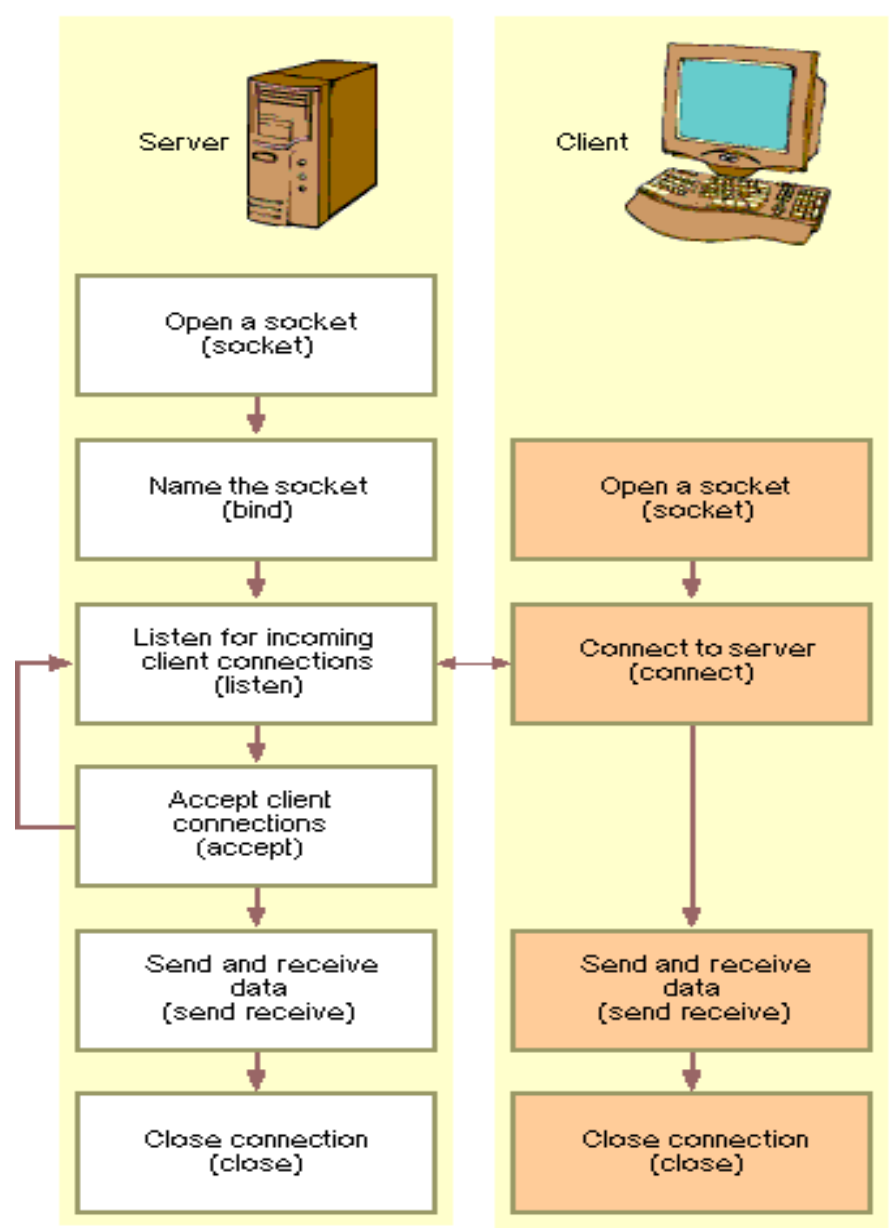

Figure 11: TCP client - server socket connection

1) The first step is to open the server socket by using AF_INET for the address format and SOCK_STREAM

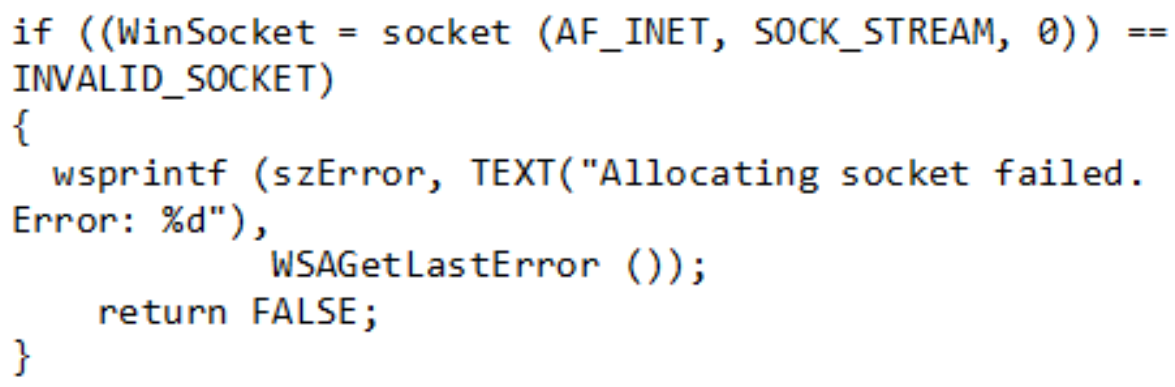

Figure 12: Socket 
2) The next step is to name the socket with the bind function using a SOCKADDR_IN structure for the address parameter.

The bind function is called to assign a name to the server socket.

The bind helps to establish the socket association. The following code example shows how to initialize SOCKADDR_IN, and then use bind.

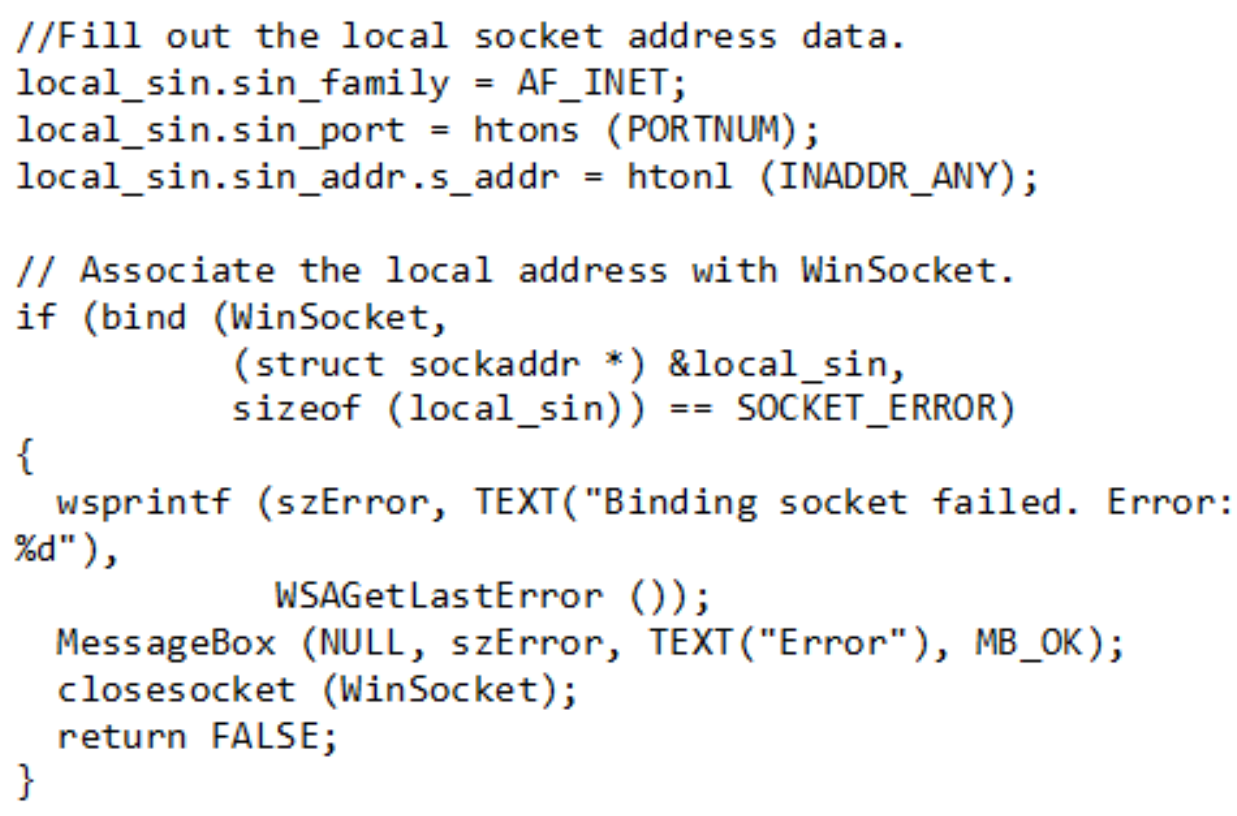

Figure 13: Bind

3) Listen for incoming client connections with listen. 
To prepare for a name association, the TCP stream server must first listen for connection requests from the TCP client with the listen function.

if (listen (WinSocket, MAX_PENDING_CONNECTS) == SOCKET_ERROR)

4) Accept a client connection with the accept function.

The tcp stream socket uses accept to accept the client connection that completes the connection. [23]

The following code example shows how to use accept.

ssock $=\operatorname{accept}($ msock, $($ struct sockaddr $*) \& f \sin , \&$ alen $)$

5) Send and receive data with a client using the send and recv functions.

After the client server socket gets connected send and recv functions can be used for exchanging the data.

recv (ClientSock, szServerA, sizeof (szServerA), 0);

send (ClientSock, "To Client.", strlen ("To Client.") + 1, 0)

However, successfully completing a call to send does not confirm that data was successfully delivered. 
6) Close the connection with the closesocket function. [23]

\subsection{TCP stream socket client application}

The following steps list how to create a TCP stream socket client connection with the server:

1) Open a stream socket using AF_INET.

2) Connect to the server using a SOCKADDR_IN structure.

3) The TCP stream client associates socket names by connecting to the stream server with connect.

The following code example shows the TCP client connecting with the server.

// Establish a connection to the server socket.

if (connect (ServerSock,

(PSOCKADDR) \&destination_sin, sizeof $($ destination_sin $))==$ SOCKET_ERROR)

4) Exchange data with a server, using the send and recv functions.

5) Close the connection with closesocket [23]. 


\section{Encryption:}

To make the connection more secure, data is encrypted before sending to the server from client side. AES is a standard cryptographic algorithm wherein a symmetric key encryption standard is adapted. This standard consists of three block ciphers as: AES128, AES-192 and AES-256. Each of the cipher has a key size of 128, 192, and 256 bits, respectively. The AES algorithm executes number of repetitions in order to transform plaintext to cipher text. Each round comprises of following steps:

1) Key Expansion step is derived from the cipher key using Rijndeal's schedule.

2) In the Initial round, each byte is combined with the round key using bitwise XOR.

3) In the Rounds step, various steps like adding SubBytes according to the look up table, shifting rows, mixing columns and adding AddRoundKey are performed.

4) Final Round: In final round above steps of adding subtypes, shifting rows and AddRoundKey are repeated without mixing the columns. 


\subsection{AES algorithm:}

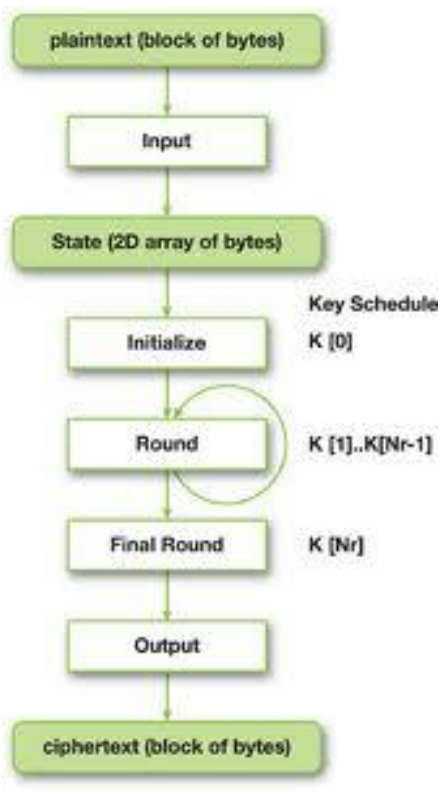

Figure 14: AES algorithm [17]

To perform the AES encryption, evp.h, openssl libraries is used to implement the following main function of encryption and decryption. 


\subsection{Encryption:}

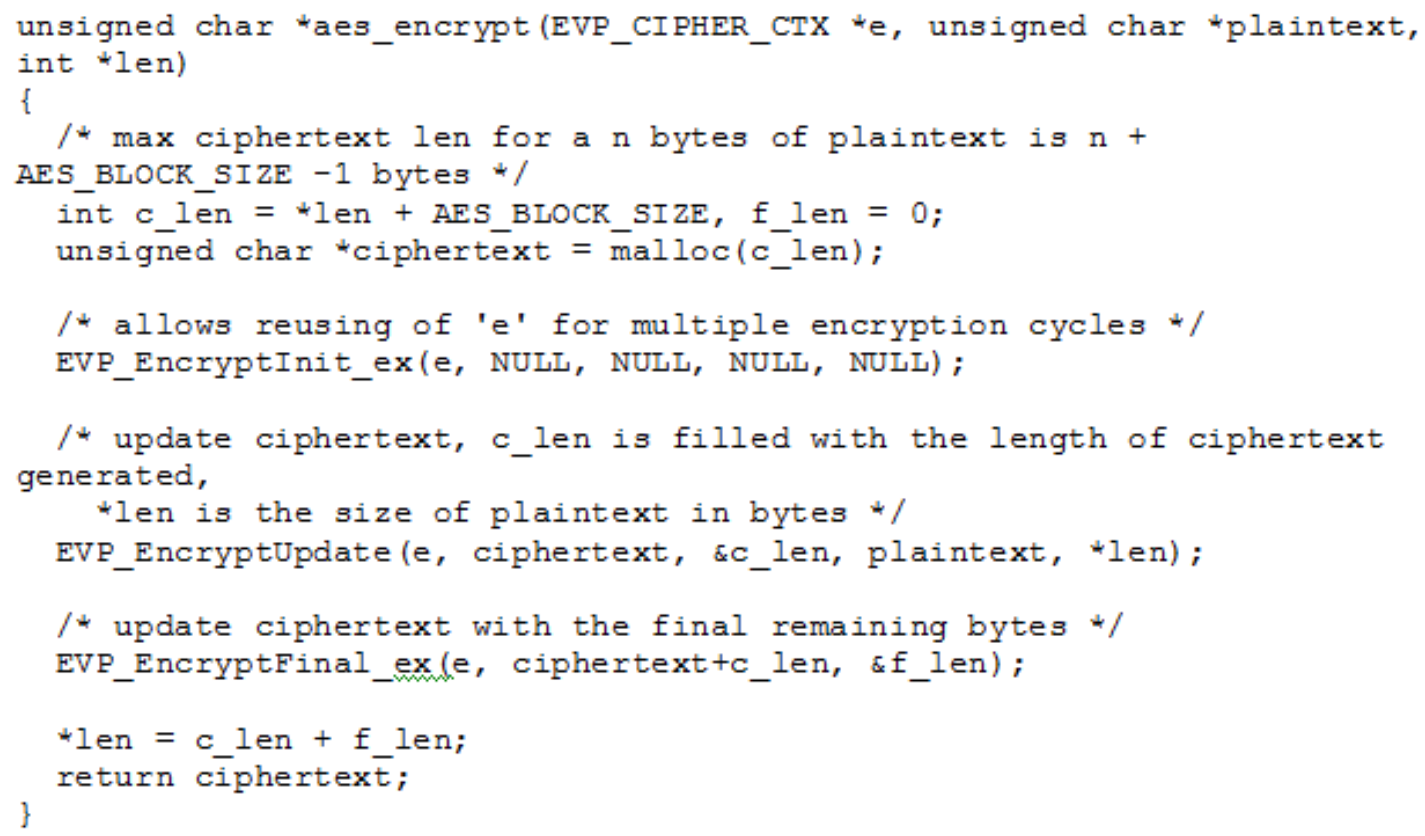

Code 8: AES encrypt 


\subsection{Decryption:}

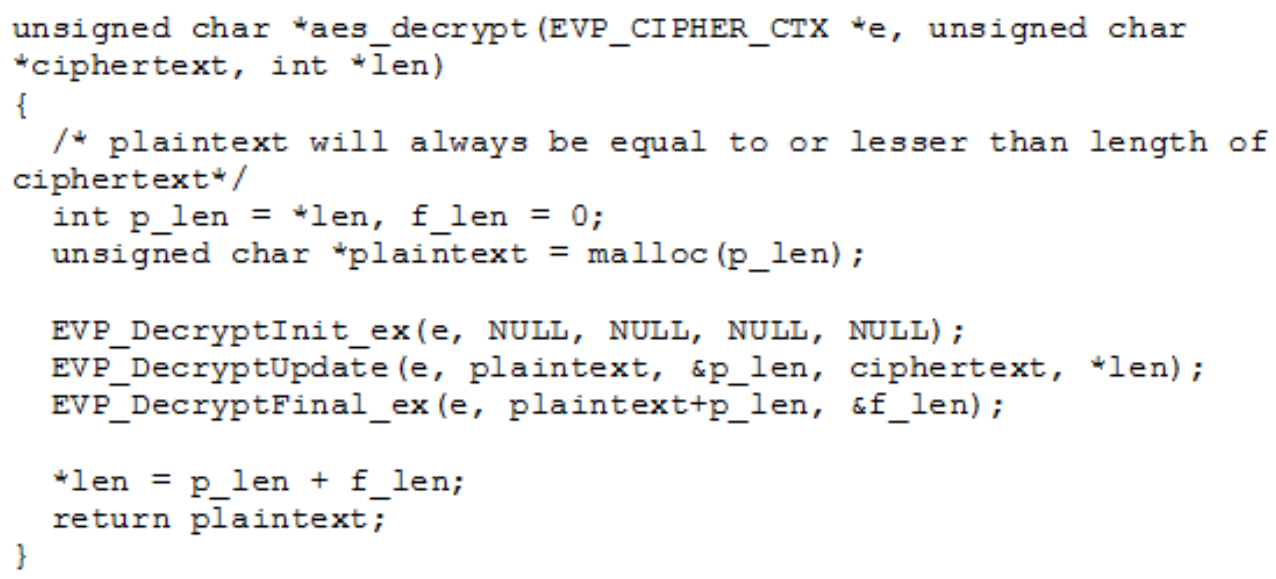

Code 9: AES decrypt 


\section{Test and results}

A secure client server connection is established. The AES standard cryptography algorithm is used to protect the data transferred from client to server using the TCP socket connection. The following shows the results obtained performing test on the tool:

1) The snapshot below shows the initialized server socket connection.

\begin{tabular}{|c|c|c|}
\hline 圆 & namrata@fineg02: /server & 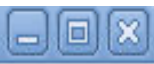 \\
\hline File Edit View Terminal & Help & \\
\hline Completed connections & $: \theta$ & A \\
\hline Total byte count & $: \theta$ & \\
\hline -. Mon Apr 18 15:49:01 & 2011 & \\
\hline Current connections & $: 0$ & \\
\hline Completed connections & $: \theta$ & - \\
\hline Total byte count & $: 0$ & \\
\hline -. Mon Apr 18 15:49:06 & 2011 & \\
\hline Current connections & $: 0$ & \\
\hline Completed connections & $: 0$ & \\
\hline Total byte count & $: 0$ & \\
\hline -.- Mon Apr 18 15:49:11 & 2011 & \\
\hline Current connections & $: 0$ & $\equiv$ \\
\hline Completed connections & $: 0$ & \\
\hline Total byte count & $: 0$ & \\
\hline -. Mon Apr 18 15:49:16 & 2011 & \\
\hline Current connections & $: \theta$ & \\
\hline Completed connections & $: 0$ & \\
\hline Total byte count & $: 0$ & \\
\hline
\end{tabular}

Figure 15: The server is started and socket is opened for the client to send the data 
2) The time taken for the encrypting a single data block with a size of 256 bytes is less than 2 msec.

3) The client sends the data after testing if the network is free to send the data without interrupting the user usage by checking the condition if IN and OUT defects; and IN and OUT errors are less than 2, the data packets are transferred from the client to server.

In figure 17, the details about the active network interface are available on the client side as well as details like network interface speed, number of data bytes end and received along with IN and OUT errors and discards. It also shows the data blocks that get transferred from client to server. 


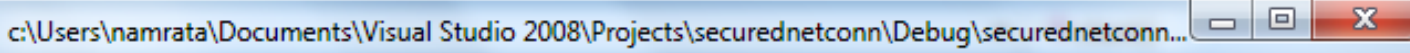

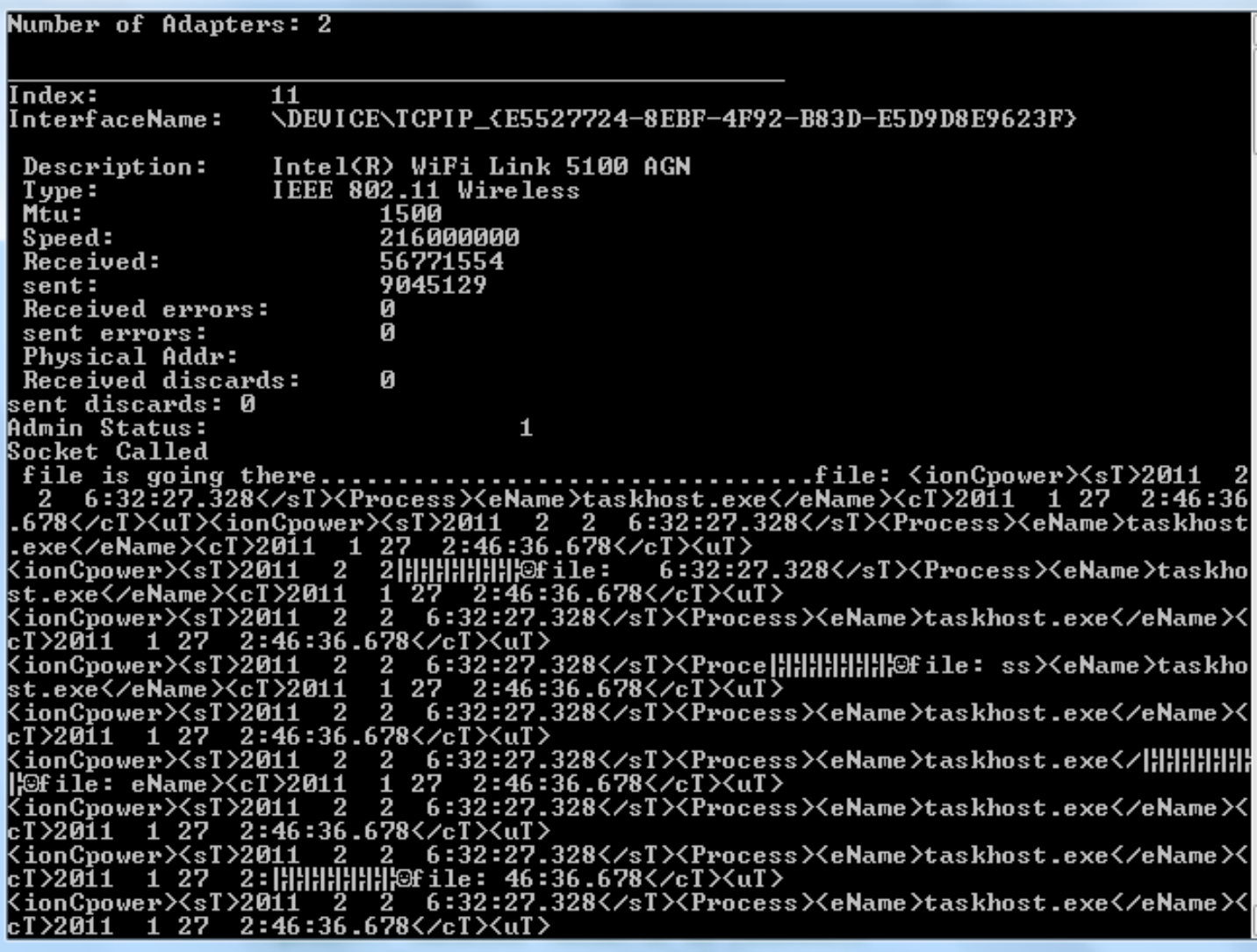

Figure 16 : Client

4) The data is received on the server side and then decrypted. The decryption time on the server side for each block of 256 bytes takes less than $\mathbf{2}$ msec.

5) Figure 18 shows the data packets received on the server. These data packets are stored at the server side for multi-client architecture and dumped into MYSQL database for the further analysis purpose after performing decryption at the server side. 
File Edit View Terminal Help

$\begin{array}{ll}\text { Current connections } & : 0 \\ \text { Completed connections } & : 0 \\ \text { Total byte count } & : 0\end{array}$

Socket Connection EastablishedWell Morris p_len $256 \mathrm{f}$ len $\theta$ $<$ ionCpower $><\mathrm{sT}>201122 \quad 6: 32: 27.328<$ /sT $><$ Process $><$ éName $>$ taskhost. exe $</$ eName $><$ CT $>20111272: 46: 36.678</$ CT $><$ UT $><$ ionCpower $><$ ST $>2011 \quad 2 \quad 2 \quad 6: 32: 27.328</$ ST $><P$ rocess $><$ eName $>$ taskhost. exe $</$ eName $<<$ CT $>2011 \quad 127 \quad 2: 46: 36.678</$ cT $><$ uT $>$ $<$ ionCpower $><$ ST $>2011 \quad 2 \quad 2$ Well Morris p_len $256 \mathrm{f}$ _en 0

$6: 32: 27.328</$ sT $><$ Process $><$ eName $>$ taskhost. exe $</$ eName $><$ C $>2011 \quad 1 \quad 27 \quad 2: 46: 36.67$ $8</ c T><U T>$

$<$ ionCpower $><\mathrm{sT}>201122 \quad 6: 32: 27.328</ \mathrm{sT}><$ Process $><$ eName $>$ taskhost. exe $</$ eName $><$ cT $>20111272: 46: 36.678</$ CT $><$ UT $>$

$<$ ionCpower $><$ sT $>2011 \quad 2 \quad 2 \quad 6: 32: 27.328</$ sT $><$ Proce

Well Morris p_len $256 \mathrm{f}$ len $\theta$

ss $><$ eName $>$ taskhost. exe $<\overline{\text { eName }><c T>2011} 127 \quad 2: 46: 36.678</$ cT $><$ uT $>$

$<$ ionCpower $><\mathrm{sT}>2011 \quad 2 \quad 2 \quad 6: 32: 27.328<$ sT $><$ Process $><$ eName $>$ taskhost. exe $</$ eName $><$ cT $>20111272: 46: 36.678</$ cT $><$ UT $>$

$<$ ionCpower $><$ ST $>201122 \quad 6: 32: 27.328</$ sT $><$ Process $><$ eName $>$ taskhost.exe $</$

Well Morris p_len $256 \mathrm{f}$ len 0

eName $><\mathrm{cT}>201 \overline{1} \quad 1 \quad 27 \quad 2: 46: 36.678</ \mathrm{cT}><\mathrm{UT}>$

$<$ ionCpower $><\mathrm{sT}>2011 \quad 2 \quad 2 \quad 6: 32: 27.328<$ sT $><$ Process $><$ eName $>$ taskhost. exe $<$ /eName $><$ cT $>2011 \quad 127 \quad 2: 46: 36.678</$ cT $><$ UT $>$

Figure 17: Received data

6) The network bandwidth utilization when sending the 10 packets of data through the network is around $2.0 \%$ during its peak - which is shown in figure 18. 


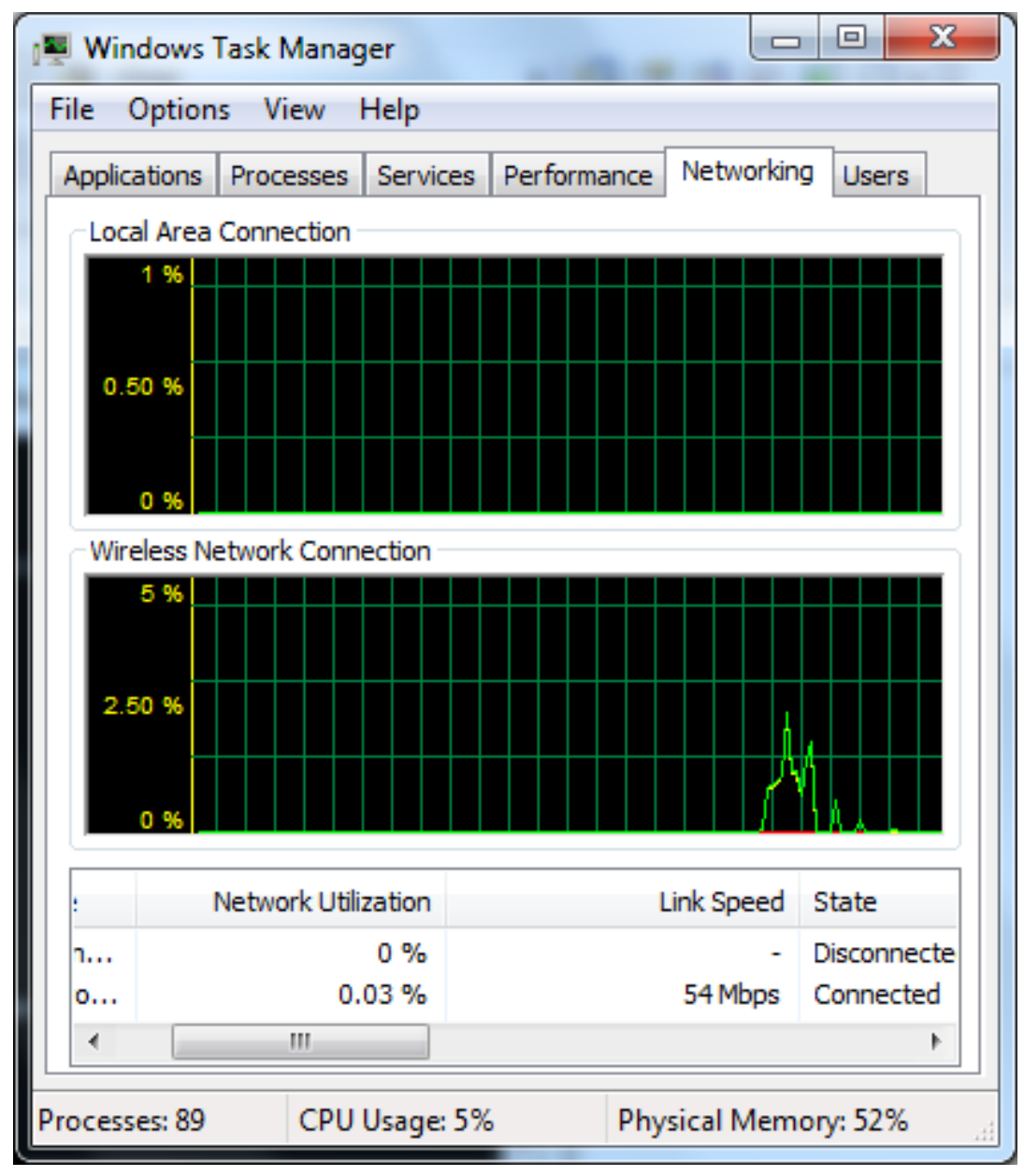

Figure 18: Network utilization

7) Figure 19 shows the CPU utilization while sending the data over the network is between $2-16 \%$. 


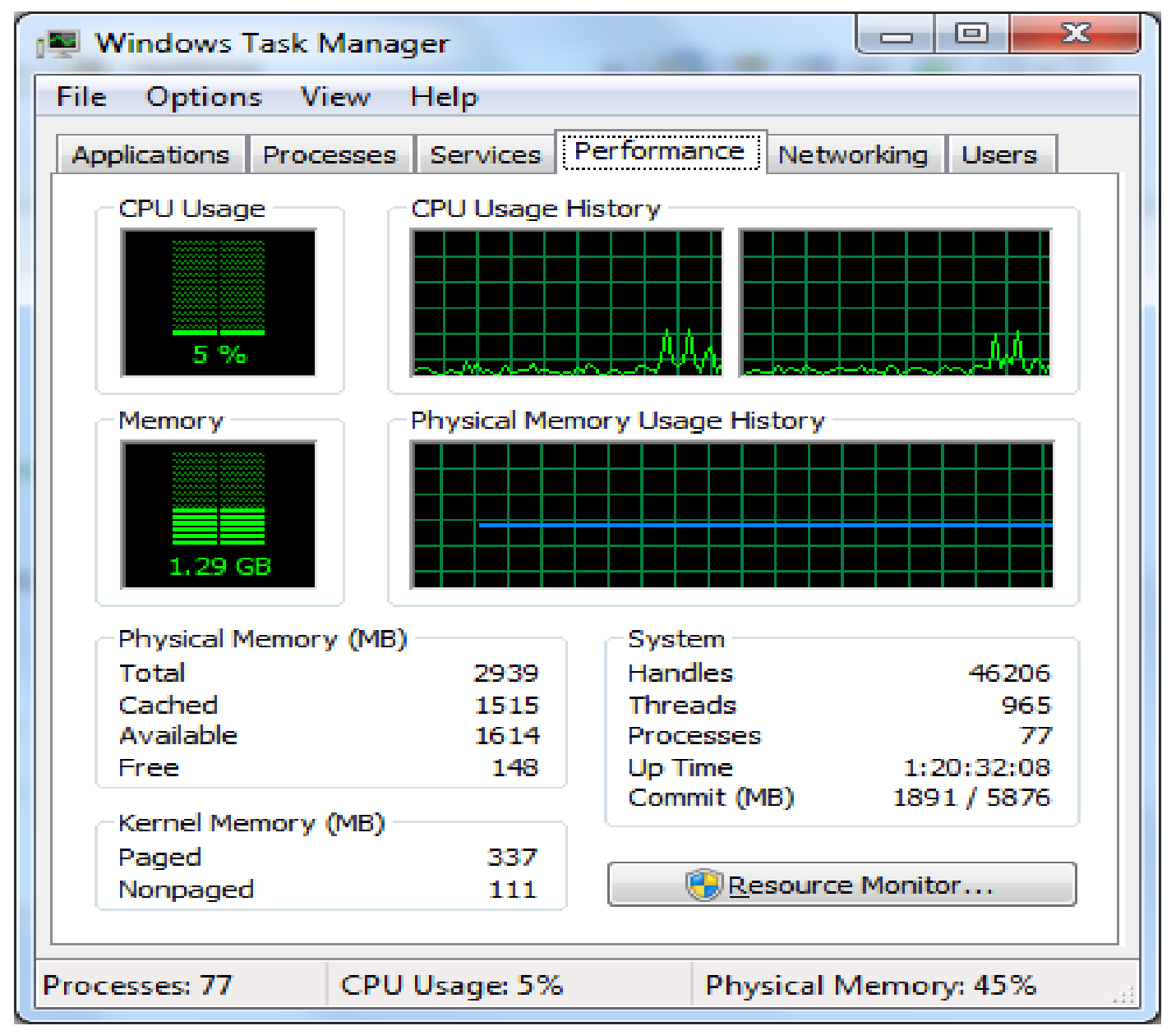

Figure 19: CPU Utilization

The feedback will be provided to each user after analyzing the data for optimizing his/her usage and reducing the energy waste. 


\section{Conclusion}

The data collection and secure transmission by utilizing the network to its optimum is successfully accomplished for further experimental purposes. Hence, iGreen, the research project, at the intersection of technology and behavioral science, will yield insights on user behavior as well as the incorporation of user feedback as part of laptop design for energy

efficiency. 


\section{Future Work}

Enhancements are required in three different areas in this project which are explained as below:

1) Encryption key management: Public key cryptography algorithm, AES, is used in this project. Currently, the project works for a single client only. In future, multiple clients will access this application making it necessary to generate multiple private keys, one for each client for performing cryptography.

2) Error recovery in control system: There is no error recovery feature supported for the Collection and Transmission module, currently. In this model, where only single client architecture is supported, there are no chances of errors. But this might change when dealing with multiple clients. The error recovery system will be useful when such errors are generated. Thus, it is an important feature that should be supported in future.

3) Self removal of data at the end of study period: The data is analyzed on server side and feedback is provided to clients on how to improve the user behavior to save laptop energy power. After this study period, it is recommended to delete that data and uninstall the application as they consume unnecessary space. Thus, in future, cleaning the memory by removing this data and uninstalling the application should be implemented to maintain appropriate functionality. 


\section{References}

1. Roth, K. W. (2007). Roth, K. W., \& McKenney, K. (2007, January). Energy consumption by Final report to the Consumer Electronics Corporation (TIAXX Reference Report D5525). . MA: TIAX LCC: Cambridge.

2. Corportation, G. (2008, June 23). PCs in Use Pass 1 Billion Mark.

3. Webber, C. A. (2001). Field surveys of office equipment operating patterns, $L B N L$ Paper $L B N L-46930$. Retrieved from http://escholarship.org/uc/item/3r7480zm

4. Roth, K.W. (2002). Energy consumption by office and telecommunications equipment in commercial buildings: Volume I (Arthur D. Little Reference No. 72895-00). Cambridge, MA: Arthur D. Little, Inc.

5. Kawamoto, K.K. (2001). Electricity used by office equipment and network equipment in the U.S.: Detailed report and appendices. Berkeley, CA: Lawrence. Berkeley, CA: Lawrence Berkeley National Laboratory: (LBNL Paper LBNL45917).

6. Collins, R. (2007, September). Analysis of the potential for minimum energy performance standards for computers and monitors. Umina Beach, NSW 2257, Australia: Punchline Energy.

7. Ranganathan, P.G. (2006). Energy-aware user interfaces and energy-adaptive displays, Computer, 39(3), 31- 38.

8. Iyer, S. L. (2003). Energy-adaptive display system designs for future mobile environments. The First International Conference on Mobile Systems, Applications, and Services (MobiSys 2003) Proceedings, (pp. 245-258).

9. Condor High Throughput Computing. (n.d.). Retrieved April 2011, from Idle Machine Statistics: http://www.cs.wisc.edu/condor/goodput/idle.html

10. Purchase, H. A.-K. (2008). Information Visualization. (Vol. Volume 4950/2008). 
11. Darby. OP cit.

12. Fischer, C. ( June 4-5, 2007.). Consumer feedback: A helpful tool for stimulating electricity conservation. Cases in Sustainable Consumption and Production: Workshop of the Sustainable Consumption Research Exchange (SCORE!) Network. Paris, France,.

13. Fischer, C. (4-9 June 2007). Influencing electricity consumption via consumer feedback. A review of experience. ECEEE 2007 Summer Study.

14. Fogg, B.J. (2002). Persuasive technology: Using computers to change what we thing and do. San Francisco: Morgan Kaufmann.

15. van Houwelingen, J.H. (1989). The effect of goal-setting and daily electronic feedback on in-home energy use.. The Journal of Consumer Research.

16. Midden, M. \&. Op cit.

17. AES encryption and decryption. (2011). Retrieved April 2011, from Developer Zone: http://http.developer.nvidia.com/GPUGems3/gpugems3_ch36.html

18. bandwidth. (2011). Retrieved April 2011, from About.com: http://compnetworking.about.com/od/speedtests/g/bldef_bandwidth.htm

19. Morris Jones, B.W. (2010). Pilot Study of College student Laptop Usage for Energy Efficiency. San Jose, California.

20. Optimal Transfer Algorithm. (2011). Retrieved April 2011, from CommServer: http://www.commsvr.com/Howitworks/Technologie/Optimization/OTA.aspx

21. Quality of service. (2011). Retrieved April 2011, from Wikipedia: http://en.wikipedia.org/wiki/Quality_of_service

22. Resource Availability Troubleshooting Getting Started Guide. (2011). Retrieved April 2011, from MSDN website: http://technet.microsoft.com/enus/library/dd883276(v=ws.10).aspx

23. Solworth, M. R. (2004, September 24). Socket Programming in $\mathrm{C} / \mathrm{C}++$. Retrieved April 2011, from http://www.rites.uic.edu/ solworth/sockets.pdf

24. Windows Task Manager. (2011). Retrieved April 2011, from Wikipedia: http://en.wikipedia.org/wiki/Windows_Task_Manager 
25. Caruso, J. B. (2009). The ECAR Study of undergraduate students and information technology 2009. Educause. (Mahesri)

26. Mahesri, A. \&. Power consumption breakdown on a modern laptop In B. Falsafi \& T. N. Vijaykumar (Eds.), Power-aware computer systems. Berlin:: SpringerVerlag.

27. Bardini, T. \&. (1995). The social construction of the personal computer user. Journal of Communication.

28. Jain, D. A. ( January 2000). Statistical Pattern Recognition: A Review. IEEE Transactions on Pattern Analysis and Machine Intelligence, Vol. 22, No. 1,.

29. Webb, A. (2002). Statistical Pattern Recognition,. John Wiley \& Sons, 2002, 2nd Edition, .

30. Process Status API. (2011). Retrieved April 2011, from MSDN Library: http://msdn.microsoft.com/en-us/library/ms684884(v=vs.85).aspx

31. IP Helper. (2011). Retrieved April 2011, from MSDN Library: http://msdn.microsoft.com/en-us/library/aa366073(v=vs.85).aspx

32. MIB Structures. (2011). Retrieved April 2011, from MSDN Library: http://msdn.microsoft.com/en-us/library/aa366889(v=VS.85).aspx

33. Condor High Throughput Computing. (n. d.).Idle Machine Statistics http://www.cs.wisc.edu/condor/goodput/idle.html

34. Snowball sampling. (2011). Retrieved from Wikipedia:

http://en.wikipedia.org/wiki/Snowball_sampling 